Review

\title{
Current Situation and Perspectives of Fruit Annonaceae in Mexico: Biological and Agronomic Importance and Bioactive Properties
}

\author{
Luis M. Hernández Fuentes 1,*(D), Efigenia Montalvo González 2,*(D), Maria de Lourdes García Magaña ${ }^{2}$, \\ Luis M. Anaya Esparza ${ }^{2,3} \mathbb{D}^{\text {, }}$, Yolanda Nolasco González ${ }^{1,2} \mathbb{D}$, Zuamí Villagrán ${ }^{4} \mathbb{D}$, Sughey González Torres $4 \mathbb{D}^{\mathbb{D}}$, \\ José Joaquín Velázquez Monreal ${ }^{5, *}$ and David Antonio Morelos Flores ${ }^{2}$
}

1 Instituto Nacional de Investigaciones Forestales, Agrícolas y Pecuarias, Santiago Ixcuintla 63300, Nayarit, Mexico; nolasco.yolanda@inifap.gob.mx

2 Laboratorio Integral de Investigación en Alimentos, Tecnológico Nacional de México/Instituto Tecnológico de Tepic, Tepic 63175, Nayarit, Mexico; mgarciam@ittepic.edu.mx (M.d.L.G.M.); luis.aesparza@academicos.udg.mx (L.M.A.E.); daanmorelos@ittepic.edu.mx (D.A.M.F.)

3 División de Ciencias Agropecuarias e Ingenierías, Centro Universitario de los Altos, Universidad de Guadalajara, Tepatitlán de Morelos 47620, Jalisco, Mexico

4 División de Ciencias Biomédicas, Centro Universitario de los Altos, Universidad de Guadalajara, Tepatitlán de Morelos 47620, Jalisco, Mexico; blanca.villagran@academicos.udg.mx (Z.V.); sgonzalez@cualtos.udg.mx (S.G.T.)

check for

Citation: Hernández Fuentes, L.M.

Montalvo González, E.; García

Magaña, M.d.L.; Anaya Esparza, L.M.; Nolasco González, Y.; Villagrán, Z.; González Torres, S.; Velázquez Monreal, J.J.; Morelos Flores, D.A. Current Situation and Perspectives of Fruit Annonaceae in Mexico: Biological and Agronomic Importance and Bioactive Properties. Plants 2022, 11, 7. https://doi.org/ $10.3390 /$ plants 11010007

Academic Editors: Kazuo N. Watanabe, Jorge Cadena-Iñiguez, Akiko Hashiguchi and Beatriz Ramos Solano

Received: 14 November 2021 Accepted: 13 December 2021 Published: 21 December 2021

Publisher's Note: MDPI stays neutral with regard to jurisdictional claims in published maps and institutional affiliations.

Copyright: (C) 2021 by the authors. Licensee MDPI, Basel, Switzerland. This article is an open access article distributed under the terms and conditions of the Creative Commons Attribution (CC BY) license (https:// creativecommons.org/licenses/by/ $4.0 /)$.
5 Instituto Nacional de Investigaciones Forestales, Agrícolas y Pecuarias, Tecomán 28925, Colima, Mexico

* Correspondence: hernandez.luismartin@inifap.gob.mx (L.M.H.F.); emontalvo@ittepic.edu.mx (E.M.G.); velazquez.joaquin@inifap.gob.mx (J.J.V.M.)

\begin{abstract}
The Annonaceae family is one of the oldest angiosperms. The genus Annona is the one with the most species and, together with Asimina, the only ones that contain edible fruits. In the last 10 years, interest in these fruit species has increased, mainly due to their nutritional properties and their application in the treatment of human diseases. Mexico is the center of origin for most of them. However, at present much of the basic agronomic information, postharvest handling of the fruits, and their potential as new crops for areas with poor soils in organic matter or semi-dry climates is unknown. It is considered that these custard apple species may be an option to change towards instead of crops that have lost profitability and sustainability. A review of the current state of knowledge in different areas of the species A. muricata, A. macroprophyllata, A. reticulata, A. squamosa, and $A$. cherimola was carried out and to focus research efforts on the topics of greatest interest and on those where is required to achieve a sustainable production and use of these resources in Mexico. However, knowledge about the cultivation and potential uses of these species is needed to increase their commercialization; the integration of interdisciplinary and interinstitutional groups is required.
\end{abstract}

Keywords: Annonaceae; Annona species; postharvest technologies; nutritional quality; bioactive compounds; biological activity; perspectives

\section{Introduction}

The Annonaceae family is one of the oldest angiosperms. In the analysis of fossils of leaves, seeds, fruits, and pollen, it is estimated that it had its origin in the late Cretaceous period [1]. The Annonaceae family is significant for ecological, evolutionary, and economic reasons [2]. Worldwide, the Global Biodiversity Information Facility (GBIF) database lists 162 genera, and 3049 species of the Annonaceae family, distributed mainly in the tropical and subtropical region of Central and South America, Africa, Asia, and Australia [3]. On the other hand, the World Checklist of Vascular Plants (WCVP) lists 111 genera with 2444 accepted species [4]. The most representative genera of the family are Annona with 215 species, Goniothalamus with 147 species, Guatteria with 314 species, Polyalthia with 
153 species, and Uvaria with 247 species, and Xylopia with 216 species [3], which together represent $42.3 \%$ of the species of this family.

In this context, in North America, the Integrated Taxonomic Information System (ITIS) includes 14 genera and 35 species. While the database of the Sistema Nacional de Información de la Biodiversidad (SNIB) mentions the presence in Mexico of 22 genera and 73 species, of these, 19 belong to the genus Annona [5]. Only the genera Annona and Asimina contain species that produce edible fruits; most of these species are found in Annona (A. cherimola Mill., A. squamosa L., A. muricata L., A. cherimola x A. squamosa, A. reticulata L., A. macroprophyllata Donn. Sm., A. glabra L., and A. purpurea Moc. \& Sessé ex Dunal, while only A. triloba is present in Asimina [6].

It is estimated that the genus Annona originated approximately 66 million years ago in the tropics and subtropics of America, Southeast Asia, and Africa [7,8]. A. cherimola, A. squamosa, A. macroprophyllata (= A. diversifolia), and A. muricata are native to Central America, and were distributed by humans to other countries [6,9]. A. muricata, A. reticulata, A. squamosa, and $A$. cherimola may have been cultivated by the Mayans for at least 3400 years B.C., to which they made selections during their cultivation [10]. On the other hand, it is believed that $A$. reticulata is native to western India and was later carried to Central America [11]. A. cherimola is a species cultivated mainly in the tropics and subtropics of America. Unlike the other fruit species of Annona, it requires a temperate climate; it is cultivated in the United States, Mexico, Spain, Australia, Guatemala, Ecuador, and Peru [6,8], Spain being the main producer with 3600 hectares. In Mexico, its development and agronomic knowledge are still limited; 36 hectares have been established, with a production of 247.3 tons [12]. Of the five fruit species of Annona, A. muricata currently presents greater agronomic development and established surface. It is cultivated mainly in Mexico, Venezuela, Ecuador, Colombia, and Brazil in areas with a warm climate from sea level to an altitude of $800 \mathrm{~m}$. It is native to Central America [1,9]. Currently, Mexico and Brazil are the main producers, and together they maintain around 8000 cultivated hectares $[12,13]$. On the other hand, A. reticulata and A. macroprophyllata are still species with little or no cultivation development. Both are currently in a semi-cultivated state in backyard orchards or in the wild in tropical and warm regions in the Mexican Pacific states. A. macroprophyllata is native to Mexico. This species was a fruit known and consumed by the Aztecs before the arrival of the Spanish, who brought it to Europe in the year 1570 [14]. It is mainly distributed in the dry tropic region in the states of Guerrero and Colima [15]. It is commercialized in local markets; the fruits are collected in wild areas, from backyard trees, or established on the banks of other crops, such as corn, and mainly grazing lands. Efforts have been made to identify and characterize phenotypes of this species with outstanding adaptive and agronomic characteristics, highlighting the collections and characterizations of Ballesteros in the Tierra Caliente region, in the state of Guerrero [16-18]. It tolerates soil conditions with low levels of nutrients, drought, and high temperatures, which makes it an interesting alternative species to grow in places with these conditions and contributes to the economy of low-income families [16]. A. reticulata is found in the wild in Mexico; however, in other countries such as India, Brazil, Peru, Bangladesh, Pakistan, and the United States, it is found in small areas of cultivation, in backyards, and in the wild from where it is collected for its consumption and sale in local markets [11]. A. squamosa is a little better-known species; it is cultivated in Central America, Mexico, India, and northwest Brazil $[12,19,20]$. It is distributed in tropical and subtropical countries in warm and semiwarm places from $200 \mathrm{~m}$ to $1300 \mathrm{~m}$ above sea level [21]. Brazil is the main producer, with around 10,000 hectares cultivated in the northwest [13].

The use of fruit Annonaceae is wide and varied; these fruits are consumed principally fresh. The processed fruits can be found in the market as different anonas-derived products such as ice cream, flakes, juice, nectar, jelly, yogurt, puree, milkshakes, syrup, jam, alcoholic beverages, desserts, and extracts of active compounds with medicinal properties [22]. However, during the last two decades, these Annona species have been the subject of numerous and diverse studies mainly due to their biological properties (ecology) 
and applications in the fields of medicine (various pathologies), nutraceuticals (antioxidants, minerals, and fiber), metabolomics (acetogenins and other insecticidal, fungicidal, and bactericidal compounds), and agriculture (alternative crops, molecular characterization, germplasm collection, characterization, plant breeding, and obtaining new varieties), among others [23].

Due to their genetic characteristics and biotechnological potential, interdisciplinary research groups have been formed in countries such as Spain, France, the United States, Japan, Brazil, Argentina, Colombia, and Mexico [24], and recently India and Bangladesh, as highlighted by numerous publications. In Mexico, the National Network of Anonaceas (NNA) was formed in 2002, made up of researchers from different institutions and disciplines of knowledge, with an emphasis on the study of the species A. muricata, $A$. cherimola, A. squamosa, $A$. diversifolia, $A$. reticulate, $A$. purpurea, and related wild genera that, faced with the increasing threat of environmental deterioration, are at risk of genetic erosion and gradual loss. The main objectives are the generation of knowledge for in situ and ex situ conservation, use and enhancement, and capacity building in these species [25]. Although some activities have been carried out in the NNA, the lack of budget support has limited the monitoring and fulfillment of these objectives. Currently, this network is part of the subcommittee on genetic resources, which in turn is coordinated by the Sectoral Committee on Genetic Resources for Food and Agriculture, whose legal basis is on the Agreement by which the Sectoral Committee on Genetic Resources for Food and Agriculture is created. The objective of this Committee is to promote the conservation, management, fair and equitable distribution of benefits, and sustainable use of genetic resources, through inter-institutional and interdisciplinary coordination in the sector [26]. Similarly, specialist meetings have been held with presentations of research results in different areas of knowledge in the Annonaceae group [24,25].

Considering the above, the objective of this review is to gather the current information generated in these Annona species, delimit the frontier of knowledge, and propose actions in the short, medium, and long term for the NNA. The JSTOR, ResearchGate, Google Scholar, Scopus databases and national production statistics, as well as technical publications from research institutes and centers were reviewed.

It is interesting to observe how the development of research in these Annona species has been, on the one hand, the basic agronomic knowledge for their commercial production, and postharvest management technologies and obtaining derived products is still limited. They have made progress only in some areas of the production process and postharvest handling. However, in topics such as phytochemistry, nutraceutical uses, and applications in different pharmacological areas, important research contributions have been constructed in several countries.

\section{Agronomic Knowledge}

Of the species of fruit custard apples or soursop, A. muricata is the one with the greatest development in its agronomic knowledge in Mexico. It has the largest cultivated area and production [12]. In Mexico, clonal varieties of $A$. muricata (breeder titles: Guanay-1, Guanay-2, and Guanay-3) have been characterized and registered [27]. However, most of the current agronomic knowledge is generalized. More specific studies are required, such as adaptation in different environments, generation of varieties with higher yields and tolerance to pests and diseases, studies of phenology and nutritional requirements, planting density and formation pruning, collection, morphological and genetic characterization, and genotype conservation. Currently, there is greater knowledge in different areas of biology, agronomy, and biotechnology in this species. Its agronomic management can be consulted in [18,28-32]. Some specific work has been carried out which contributes to optimal production. The management of the main pests and disease problems can be consulted in [28,33-36]. Similarly, important advances have been made in the collection, description, and selection of genotypes [32,37-39], with which improvement activities could begin. 
The species $A$. cherimola is second in economic importance in Mexico. However, the cultivation area and production are smaller, with an establishment potential in central and southern Mexico [40]. Currently, it is only cultivated in Michoacán and Morelos [12]. It is found naturally in temperate and subtropical zones in the states of Morelos, Oaxaca, Michoacán, Hidalgo, Veracruz, Chiapas, Puebla, Jalisco, Guanajuato, and the State of Mexico [41]. In this context, some genotypes of $A$. cherimola have been characterized and registered, including Tonaltzintl, Metztli 34, Selección 94-33, Álvaro, Urhuata, and Lamtl 256 [27]. As with A. muricata, its agronomic knowledge in Mexico is limited, and the recommended management is generalized [42,43]. Research has been carried out in specific areas such as pollination [44], selection and characterization [45,46], and pests and pathogens [47-50]. The knowledge of the crop is more advanced in Spain, technology has been developed, along with a selection of varieties for their best use [51-55].

On the other hand, the species A. macroprophyllata, A. reticulata, and A. squamosa have less development and knowledge in Mexico. These species do not have a breeder's title and registration in National System of Genetic Resources (SNICS- acronym in Spanish), and there is less agronomic knowledge about them. However, they have a potential for cultivation as an alternative or complementary option to the production of tropical fruit trees that have lost profitability. A. macroprophyllata, commonly known as ilama, is found naturally from Colima in Mexico to El Salvador [14]. At present, there is no knowledge of any cultivated area, as its trade is local, and all of the fruit comes from the collection of trees that are found naturally on the edge of pastures or orchards and in backyards. The first studies for its knowledge in Mexico were carried out by members of the National Network Anonaceae (NNA). In the communities of Tierra Caliente, in the Balsas region of southern Mexico, this fruit is most widely known. Its production is rustic; it presents tolerance to high temperatures, drought, and soils poor in nutrients and organic matter [16]. A research group from the Technological Institute of Ciudad Altamirano, Guerrero, as members of the NNA, has managed to identify and describe the physical-chemical characteristics of 24 types of ilama, of which 146 accessions were collected for propagating and promoting its conservation and cultivation. It is estimated that there are approximately 100 types of ilama in this region [16]. The morphological description and genomic identification of the most representative types of ilama can be consulted in the catalog Diversity in the ilamas of Tierra Caliente del Balsas, Mexico [56]. In addition to the above, more specific studies have been carried out to identify its chemical composition and antioxidant activity [57]. This species represents an interesting option due to its adaptation and pleasant flavor, with development potential for the semi-dry climate regions in the states of Colima, Guerrero, Michoacán, and Oaxaca.

A. reticulata is known as sugar apple, custard apple, or ox heart custard apple; native to India and Central America, there are no reports about the large of cultivated areas. It is mainly found naturally in tropical and subtropical regions, and only a few small areas are cultivated in south Florida, Bahamas, Bangladesh, Pakistan, and some parts of India [58,59]. In Mexico, there are no reports of its cultivation. It is found in its natural state in the Pacific region, from southern Sinaloa to Chiapas; it is not cultivated commercially, and its consumption is local in the production season. It has been observed in Nayarit, Jalisco, and Colima that the fruits are collected in the months of March to May; their consumption is fresh, and presents a great phenotypic variety, which would imply greater selection, characterization, and possible use as a crop in these areas. Its potential for use and research in medicine has great potential; numerous studies have been carried out on its chemical constitution and pharmacology, finding satisfactory results [60-64]. Regarding A. squamosa, known as saramuyo, it has a cultivated area of 50.5 hectares in Mexico, with a production of 431 tons. Its cultivation is recent, and it is located mainly in the south of the country, in the state of Yucatán. [12]. It is distributed naturally from the tropics in Mexico to Bolivia [21,65], with Brazil being the main producer [13]. Until a few years ago, in Mexico, the saramuyo was only found to be naturally occurring; however, recent research has contributed to a better understanding of this species. Similar to other Annona species, in saramuyo, there 
are numerous studies on chemical composition, evaluation, and use of its pharmacological properties [66-68], and little research on the selection, characterization, improvement, and agronomic management. General recommendations for its knowledge and agronomic management can be consulted in the Manual of Propagation and production of saramuyo (Annona squamosa L.) [69]. However, its potential for alternative cultivation as the main activity in areas with warm and semi-dry climates and soils poor in the organic matter makes the saramuyo a species that requires more attention and research.

\subsection{Nutritional Composition}

Table 1 shows the nutritional composition and energy value of the edible part of $A$. muricata, A. cherimola, A. squamosa, and A. macroprophyllata. A. cherimola and $A$. squamosa fruits are sweeter than $A$. muricata, $A$. reticulata, and $A$. macroprophyllata. They have a low content of lipids and proteins [70-79]. Furthermore, the pulp exhibits a good quantity of dietary fiber, although $A$. muricata and $A$. cherimola fruits are more fibrous. The pulps are prominent in some vitamins $(\mathrm{C}, \mathrm{E}$, thiamin, riboflavin, and niacin) and minerals $(\mathrm{Ca}$, $\mathrm{P}, \mathrm{Mg}, \mathrm{K}$, and $\mathrm{Fe}$ ). In addition, they are very low in calories. In this context, the regular consumption of these fruits provides essential nutrients for the recommended daily intake.

Table 1. Nutritional composition of edible parts from Annona muricata, A. cherimola, A. squamosa, and A. macroprophyllata fruits.

\begin{tabular}{|c|c|c|c|c|}
\hline Parameter & A. muricata & A. cherimola & A. squamosa & A. macroprophyllata \\
\hline Total Energy (kcal) & $55.4-81.73$ & 81-102 & $92.9-97.7$ & $56.06-89.03$ \\
\hline Moisture (\%) & $80.48-83.2$ & $68.7-70.4$ & $65-75$ & $71.5-79.61$ \\
\hline Protein $(\%)$ & $0.69-1.10$ & $1.36-1.96$ & $0.7-1.89$ & $0.44-1.31$ \\
\hline Lipids (\%) & $0.20-0.97$ & $0.10-0.29$ & $0.048-0.57$ & $0.16-0.31$ \\
\hline Soluble carbohydrates (\%) & $12.50-18.23$ & $13.0-29.0$ & $20.41-25.19$ & $13.55-20.25$ \\
\hline Dietary fiber $(\%)$ & $4.83-5.76$ & $2.09-5.32$ & $0.62-1.41$ & $0.97-1.30$ \\
\hline \multicolumn{5}{|l|}{ Minerals (mg/100 g) } \\
\hline Calcium & $9.0-10.3$ & $9.0-27.14$ & $17.0-44.7$ & $0.86-31.60$ \\
\hline Phosphorous & $27.7-29.0$ & $24.0-35.20$ & 54.0 & 51.7 \\
\hline Iron & $0.64-0.82$ & $0.25-0.60$ & $0.3-1.34$ & ND \\
\hline Magnesium & 22.0 & 17 & $21.0-22.0$ & $8.0-14.01$ \\
\hline Copper & ND & ND & 0.086 & ND \\
\hline Manganese & ND & ND & 0.10 & ND \\
\hline Zinc & ND & 0.16 & 0.1 & $0.10-0.13$ \\
\hline Potassium & 320 & 288 & 142 & $335.95-347.40$ \\
\hline \multicolumn{5}{|l|}{ Vitamins (mg/100 g) } \\
\hline Vitamin C & $22.59-40.56$ & $12-6-25.43$ & $25.6-58.75$ & $1.51-13.6$ \\
\hline Vitamin E & 29 & 0.27 & ND & ND \\
\hline Thiamin & $0.11-2.10$ & $0.09-0-0.11$ & 0.10 & 0.24 \\
\hline Riboflavin & $0.05-0.2$ & $0.11-0.13$ & 0.06 & 0.30 \\
\hline Niacin & $0.21-1.52$ & $0.65-1.0$ & 0.89 & 2.18 \\
\hline Cobalamin & ND & 0.12 & ND & ND \\
\hline Refences & {$[70,71]$} & [72-74] & [75-78] & {$[76,79]$} \\
\hline
\end{tabular}

$\mathrm{ND}=$ No determined.

\subsection{Tradicional Medicine Use}

In addition to the nutritional importance of the Annona fruits, different reports have demonstrated the use of all parts from the trees of A. muricata, A. cherimola, A. squamosa, A. reticulata, and $A$. macroprophyllata in traditional medicine. Since many years ago, the native people of various cultures have consumed beverages produced from Annonaceae stem bark, roots, leaves, fruit pulp, peel, and seed for a wide range of illnesses. The preparation of beverages has been by maceration, decoction, or infusion [80]. People have consumed these products for the treatment of illnesses such as parasitic infections, 
dysentery, fever, urethritis, hematuria, and asthma, liver diseases, diarrhea, and for their anticold, antispasmodic, antisudorific, anti-depressive, and antiemetic properties [81]. Nonetheless, most biological properties of members of the Annonaceae family are attributed to the presence of diverse secondary metabolites/bioactive compounds [82].

\subsection{Bioactive Compounds Identified in Fruit Annona Species}

Tables 2 and 3 show some phytochemicals (quantitative or qualitative) identified in pulp, leaf, peel, seeds, roots, and stem bark of fruit Annona species: A. muricata, A. cherimola, A. squamosa, A. reticulata, and $A$. macroprophyllata [83-89]. These compounds include phenolic compounds, alkaloids, fatty acids, cyclopeptides, alkaloids, and acetogenins.

\subsubsection{Phenolic Compounds and Fatty Acids}

The phenolic compounds are the most abundant metabolites in Annona fruits, and they are considered the principal exogenous antioxidants. Their basic structure contains an aromatic ring with one or more hydroxyl substituents [83]. However, there are some Annona species that have not been completely characterized, and one of them is A. macroprophyllata (Table 2). In A. muricata has been identified and quantified the highest diversity of phenolic compounds from pulp, leaf, peel, and seed compared to those observed in other Annona species. Among the most significant are hydroxybenzoic acids, hydroxycinnamic acids, and flavonoids. In a similar way, phenolic compounds have been reported in the pulp and leaves of $A$. cherimola. The presence of flavonoids, for example, derivates of quercetin, gallocatechin, and kaempferol, give significant biological value to fruits as well as leaf extracts [84]. Moreover, the presence of phenolic compounds and flavonoids has been qualitatively reported in the edible part, leaf, and seed of $A$. squamosa [83-85,90-97]. Chlorogenic acid, ferulic acid, $p$-hydroxybenzoic acid, caffeic acid, gallic acid, and epicatechin are the most abundant phenolic compounds in this specie [86].

Table 2. Bioactive compounds: phenolic compounds and fatty acids from different plant components of some Annona species.

\begin{tabular}{|c|c|c|c|c|}
\hline Annona Specie & Plant Part & Bioactive Compounds & Content & References \\
\hline \multicolumn{5}{|c|}{ Phenolic compounds } \\
\hline \multirow{17}{*}{ A. muricata } & \multirow{7}{*}{$\begin{array}{c}\text { Pulp } \\
(\mu \mathrm{g} / \mathrm{g} d w)\end{array}$} & Chlorogenic acid & 12.80 & \\
\hline & & Cinnamic acid & 42.04 & \\
\hline & & Coumaric acid & 0.07 & \\
\hline & & Gallic acid & 15.86 & [83] \\
\hline & & 4-Hydroxybenzoic acid & 131.63 & \\
\hline & & Neochlorogenic acid & 72.32 & \\
\hline & & $\begin{array}{l}\text { Dicaffeoylquinic acid, caffeoylquinic acid, fisetin, } \\
\text { dihydrokaempferol-hexoside, morin, kaempferol 3-O-rutinoside, } \\
\text { kaempferol, luteolin 3'7-di-O-glucoside, myricetin }\end{array}$ & NR & [90-92] \\
\hline & $\begin{array}{c}\text { Leaf } \\
(\mu \mathrm{g} / \mathrm{g} d w)\end{array}$ & $\begin{array}{l}\text { Caffeoylquinic acid, chlorogenic acid, dicaffeoylquinic acid, } \\
\text { feruloylquinic acid, cinnamic acid, isoferulic acid, caffeic acid, } \\
\text { gallic acid, apigenin-6-C-glucoside, argentinine, catechin, } \\
\text { coumaric acid, daidzein, epicatechin, gallocatechin, genistein, } \\
\text { glycitein, homoorientin, kaempferol, kaempferol 3-O-rutinoside, } \\
\text { luteolin } 3 \text { '7-di-O-glucoside, quercetin, quercetin 3-O-glucoside, } \\
\text { quercetin 3-O- neohesperidoside, quercetin 3-O-robinoside, } \\
\text { quercetin-O-rutinoside, quercetin 3-O- } \alpha \text {-rhamnosyl, robinetin, } \\
\text { tangeretin, taxifolin }(+) \text {,vitexin }\end{array}$ & NR & {$[90-92,98]$} \\
\hline & \multirow{9}{*}{$\begin{array}{c}\text { Peel } \\
(\mu \mathrm{g} / \mathrm{g} d w)\end{array}$} & Gallic acid & 14.50 & \multirow{9}{*}{ [83] } \\
\hline & & Coumaric acid & 1.37 & \\
\hline & & Cinnamic acid & 45.51 & \\
\hline & & Caffeic acid & 43.68 & \\
\hline & & Chlorogenic acid & 32.67 & \\
\hline & & Protocatechuic acid & 150.46 & \\
\hline & & 4-Hydroxybenzoic acid & 145.98 & \\
\hline & & Syringic acid & 883.71 & \\
\hline & & Neochlorogenic acid & 78.86 & \\
\hline
\end{tabular}


Table 2. Cont.

\begin{tabular}{|c|c|c|c|c|}
\hline Annona Specie & Plant Part & Bioactive Compounds & Content & References \\
\hline \multicolumn{5}{|c|}{ Phenolic compounds } \\
\hline & & Gallic acid & 0.36 & \multirow{8}{*}[83]{} \\
\hline & & Coumaric acid & 0.07 & \\
\hline & & Cinnamic acid & 40.48 & \\
\hline & Seed & Caffeic acid & 32.62 & \\
\hline & $(\mu \mathrm{g} / \mathrm{g} \mathrm{dw})$ & Chlorogenic acid & 12.33 & \\
\hline & & Protocatechuic acid & 133.47 & \\
\hline & & Syringic acid & 780.77 & \\
\hline & & Neochlorogenic acid & 69.70 & \\
\hline \multirow{9}{*}{ A. cherimola } & Pulp & $\begin{array}{l}\text { Catechin, procyanidin (B-type) dimer isomer, } \\
\text { (epi)catechin-(epi)gallocatechin, epicatechin, derivates of } \\
\text { procyanidin trimer }\end{array}$ & NR & {$[93]$} \\
\hline & \multirow{8}{*}{$\begin{array}{l}\text { Leaf } \\
(\mathrm{mg} / 100 \mathrm{~g} \\
\mathrm{dw})\end{array}$} & Catequin & $12.42-24.5$ & \multirow{8}{*}[84]{} \\
\hline & & Quercetin 3-O-rutinoside-7-O-glucoside & $1.06-16.16$ & \\
\hline & & Epicatechin & $6.33-26.31$ & \\
\hline & & Quercetin 3-O-rutinoside-7-O-pentoside & $32.25-75.29$ & \\
\hline & & Quercetin 3-O-rutinoside & $719.53-2593.92$ & \\
\hline & & Kaempferol-3-Galactoside-7-Rhamnoside & $55.74-620.98$ & \\
\hline & & Kaempferol-3-O-glucoside & $22.44-337.09$ & \\
\hline & & Luteolin-3-Galactoside-7-Rhamnoside & $47.49-120.58$ & \\
\hline \multirow{2}{*}{ A. squamosa } & Leaf & $\begin{array}{l}\text { Quercetin, quercetin 3-O-glucoside, rutin, gallic acid, chlorogenic } \\
\text { acid, isorhamnetin, ferulic acid, kaempferol, caffeic acid }\end{array}$ & NR & {$[85,95]$} \\
\hline & Seed & $\begin{array}{l}\text { Gallic acid, } \rho \text {-hydroxybenzoic acid, syringic acid, ferulic acid, } \\
\text { ellagic acid, benzoic acid, o-coumaric acid, and salicylic acid }\end{array}$ & NR & {$[96]$} \\
\hline \multirow{10}{*}{ A. diversifolia } & \multirow{6}{*}{$\begin{array}{c}\text { Peel } \\
(\mathrm{mg} / 100 \mathrm{~g})\end{array}$} & Chlorogenic acid & 84 & \multirow{6}{*}[86]{} \\
\hline & & Ferulic acid & 34.9 & \\
\hline & & p-hydroxybenzoic & 20.90 & \\
\hline & & Caffeic acid & 6.6 & \\
\hline & & Gallic acid & 4.9 & \\
\hline & & Epicatechin & 102 & \\
\hline & \multirow{10}{*}{ Seed $(\%)$} & Oleic acid & 70.42 & \multirow{4}{*}[79]{} \\
\hline & & Palmitic acid & 16.40 & \\
\hline & & Linoleic acid & 7.97 & \\
\hline & & Stearic acid & 5.22 & \\
\hline \multirow{6}{*}{ A. muricata } & & Palmitic acid & & \multirow{6}{*}[97]{} \\
\hline & & Linoleic acid & 25.5 & \\
\hline & & Stearic acid & 1.5 & \\
\hline & & Stearic acid & 6.0 & \\
\hline & & Oleic acid & 39.5 & \\
\hline & & Linoleic acid & 27.0 & \\
\hline \multirow{7}{*}{ A. cherimola } & \multirow{7}{*}{ Seed $(\%)$} & Myristic acid & 0.05 & \multirow{7}{*}[99,100]{} \\
\hline & & Palmitic acid & 14.91 & \\
\hline & & Heptadecanoic acid & 0.21 & \\
\hline & & Stearic acid & 7.60 & \\
\hline & & Palmitoleic acid & 0.32 & \\
\hline & & Oleic acid & $35.20-43.72$ & \\
\hline & & Linoleic & $32.48-44.93$ & \\
\hline \multirow{9}{*}{ A. squamosa } & \multirow{9}{*}{ Seed (\%) } & Oleic acid & 41.9 & \multirow{9}{*}{ [87] } \\
\hline & & Palmitic acid & 14.7 & \\
\hline & & Linoleic acid & 26.6 & \\
\hline & & Stearic acid & 11.3 & \\
\hline & & Hexadenoic acid & $10-14$ & \\
\hline & & Hepatadecene-(8)-carbonic acid (1) & 29.68 & \\
\hline & & Cis-vaccenic acid & 10.39 & \\
\hline & & Heneicosanoic acid & 3.20 & \\
\hline & & 9-octadecenoic acid (Z)-,2,3-dihydroxy propyl ester & 13.33 & \\
\hline
\end{tabular}


Table 3. Bioactive compounds: cyclopeptides, alkaloids, and acetogenins from different plant components of some Annona species.

\begin{tabular}{|c|c|c|c|}
\hline Annona Species & Plant Part & Bioactive Compound & References \\
\hline & & Cyclopeptides & \\
\hline A. muricata & Seed & Annomuricatin A-C & {$[101,102]$} \\
\hline A. cherimola & Seed & Cherimolacyclopeptide E, herimolacyclopeptide F & {$[103,104]$} \\
\hline \multirow{3}{*}{ A. squamosa } & Seed & $\begin{array}{c}\text { Cyclosquamosins A-G, } \\
\text { cyclosquamosin, met-cherimolacyclopeptide B }\end{array}$ & [105-107] \\
\hline & Peel & Fanlizhicyclopeptide A-B & [108] \\
\hline & \multicolumn{3}{|c|}{ Alkaloids } \\
\hline \multirow{5}{*}{ A. muricata } & Root, bark & Anomurine, anomuricine, atherosperminine, coreximine, coclaurine & [109] \\
\hline & Stem & $\begin{array}{c}\text { Atherospermine, casuarine, } \\
\text { 2,5-dihydroxymethyl-3,4,dihydroxypyrrolidine, } \\
\text { deoxymannojirimycin, deoxynojirmycin }\end{array}$ & {$[109,110]$} \\
\hline & Leaf & $\begin{array}{l}\text { Stepharine, coclaurine, coreximine, annonaine, asimilobine, } \\
\text { 2,5-dihydroxymethyl-3,4,dihydroxypyrrolidine, } \\
\text { deoxymannojirimycin, deoxynojirmycin, swainsonine, } \\
\text { (R)-O,O-dimethylcoclaurine, annonamine, } \mathrm{R})-4^{\prime} \mathrm{O}-\text {-methylcocaurine, } \\
\text { S)-narcorydine, xylopine, N-methylcoclaurine, remerine, isoboldine, } \\
\text { isolaureline, liriodenine, reticuline, N-methylcoculaurine }\end{array}$ & [109-114] \\
\hline & Fruit & Nornuciferine, annonaine, asimilobine & {$[111,113,114]$} \\
\hline & Peel & Nornuciferin, assimilobin, anonaine, isolaureline & [83] \\
\hline \multirow[t]{2}{*}{ A. cherimola } & Leaf & $\begin{array}{l}\text { Liriodenine, anonaine, nornuciferine, } 1,2- \\
\text { dimethoxy-5,6,6a,7-tetrahydro- } 4 \mathrm{~h} \text {, dibenzoquinoline-3,8,9,10-tetraol, } \\
\text { asimilobine, pronuciferine }\end{array}$ & [84] \\
\hline & Root & Corytenchine, isocoreximine & [115] \\
\hline \multirow{3}{*}{ A. squamosa } & Leaf & $\begin{array}{l}\text { Reticuline, o-methylarmepavine, annonaine, oxophoebine, } \\
\text { lysicamine, n-methylcoclaurine, liriodenine, corydine, lanuginosine, } \\
\text { roemerine, corypalmine, sanjoinine, norlaureline, norcodeine, } \\
\text { oxalanobie, aporphine }\end{array}$ & {$[75,116-118]$} \\
\hline & Seed & $\begin{array}{c}\text { Annonaine, asimilobine, liriodenine, corypalmine, reticuline, } \\
\text { nornuciferine }\end{array}$ & [119] \\
\hline & Stem bark & Roemerolidine, $N$-nitrosoxylopine, duguevalline & [120] \\
\hline \multirow{3}{*}{ A. diversifolia } & Seed & Rolliniastatin-2, laherradurin, cherimolin-2 and liriodenine & {$[88,121]$} \\
\hline & Root, Stem bark, leaf & Liriodenine, atherospermidine, lysicamine & [122] \\
\hline & & Acetogenins & \\
\hline \multirow{5}{*}{ A. muricata } & Pulp & $\begin{array}{l}\text { Montecristin, epomuricenins A-B, epoxymurin, epomurinins A-B, } \\
\text { epomusenins A-B, annonacin, corossolone, muricatin C, } \\
\text { muricin, montanacin }\end{array}$ & [123-126] \\
\hline & Leaf & $\begin{array}{l}\text { Annocatalin, annohexocin, annomuricin A-E, annomutacin, } \\
\text { annonacin, annopentocin A-C, corossolone, gigantetronenin, } \\
\text { goniothalamicina, montanacin, muricapentocin, muricatalicin, } \\
\text { muricin, muricatalin, muricatocin A-C, murihexocin, } \\
\text { muricoreacin, solamin }\end{array}$ & {$[82,127-129]$} \\
\hline & Seed & $\begin{array}{c}\text { Ronbusticin, annomuricatin A, cohibin A-D, donhexocin, muricatenol, } \\
\text { murihexol, epomuricenins A-B, corepoxylone, Epoxyrollin B, } \\
\text { annoglaxin, annomontacin, annonacin, annoreticuin-9-one, arianacin, } \\
\text { corossolina, corossolona, gigantetrocin A-B, goniothalamicina, } \\
\text { muricatetrocin A-B, Muricatin A-D, muricin A-I, murisolina, solamin, } \\
\text { bullatacilin, bullatacin, gigantecin, annocatacin }\end{array}$ & {$[82,89,101,130-134]$} \\
\hline & Root & Cohibin A-B, montecristin, epomuricenins A-B, sabadelin, annonacin & {$[82,125]$} \\
\hline & Stem bark & Muricatin C & [124] \\
\hline \multirow{3}{*}{ A. cherimola } & Pulp & Bullatacin, annonacin & [135] \\
\hline & Leaf & Molvizarin, cherimolin-1, motrilin, annonacin, annonisin & [136] \\
\hline & Seed & $\begin{array}{l}\text { Cherimolin, dihydrocherimolin, molvizarin, motrilin, itrabin, jetein, } \\
\text { cherimolin-2, almunequin, annomolin, annocherimolin }\end{array}$ & {$[137,138]$} \\
\hline
\end{tabular}


Table 3. Cont.

\begin{tabular}{|c|c|c|c|}
\hline Annona Species & Plant Part & Bioactive Compound & References \\
\hline & Stem bark & Aromin-A, squamocin & {$[138]$} \\
\hline \multirow{3}{*}{ A. squamosa } & Leaf & Murihexocin C & [139] \\
\hline & Seed & $\begin{array}{c}\text { Annotemoyin, squamocin, annoglaxin, epoxyrolin, murisolin, neo- } \\
\text { desacetyluvaricin, squamostatin, annosquamins, bullatacin, } \\
\text { annosquacin, annosquatin, annonareticin, motrilin, solamin, } \\
\text { squadiolin, squamoxinone, squamostanin, uvarigrandin, } \\
\text { squamostolide, tripoxyrollin, uvariamicin }\end{array}$ & {$[75,140-142]$} \\
\hline & Stem bark & $\begin{array}{c}\text { 4-deoxyannoreticuin, cis-4-deoxyannoreticuin, (2-4-cis and } \\
\text { trans)-squamoxinone, annoreticuin-9-one, bullatacin, molvizarin, } \\
\text { mosin, parviflorin, squamotacin }\end{array}$ & {$[75,143]$} \\
\hline A. macroprophyllata & Seed & Laherradurin, rolliniastin-2, and cherimolin-2 & [89] \\
\hline
\end{tabular}

The fatty acids are compounds based on carbon chains with a methyl group at one end of the structure and a carboxyl group at the other end. They are predominantly hydrophobic compounds $[79,86]$. The seeds from Annona fruit are mainly valuable for their fatty acid content (Table 2). For example, the palmitic, stearic, oleic, and linoleic acids were identified as the major fatty acids of Annona seed oils. In general, the content and type of phenolic compounds and fatty acids depend on each plant part and fruit Annona species; however, their biological importance is due to their contribution to the total antioxidant activity with pharmacological activities, mainly in preventing or slowing the progress of diverse oxidative stress-related diseases when they are consumed [87].

\subsubsection{Cyclopeptides}

Cyclopeptides or cyclic peptides are polypeptides formed from amino acids organized in a cyclic ring structure [101,102]. In the seeds of some Annona species bioactive compounds named cyclopeptides have been reported that in recent years have been of much pharmaceutical interest. Annomuricin A-C has been extracted and identified from A. muricata seeds (Table 3) [89,101,102]. Two other cyclopeptides (cherimolacyclopeptide $\mathrm{E}$ and F) were obtained from $A$. cherimola seeds $[103,104]$. Moreover, seven cyclopeptides (cyclosquamosin A-G and met-cherimolacyclopeptide B) were reported in seeds, and two cyclopeptides (fanlizhicyclopeptide A and B) were found in the peel of A. squamosa [105-108]. The Annona cyclopeptides show a remarkable variety of derivatives and a wide array of bioactivities, including antiproliferative, antimicrobial, anthelmintic, and anti-inflammatory, and cytotoxicity [104].

\subsubsection{Alkaloids}

Alkaloids that have been qualitatively identified in different plant parts of the Annona species are the isoquinoleic alkaloids. The most frequently found include aporphine, protoberberines, benzyloquinoleins, and the bis-benzylisoquinolein dimers [83]. In the leaves, seeds, stem bark, root, and peel from A. muricata, A. cherimola, A. squamosa, and A. diversifolia, anomurine, anomuricine, atherosperminine, coreximine, coclaurine, corytenchine, isocoreximine asimilobine, annonaine, corypalmine, liriodenine, nornuciferine, reticuline have been reported, among many others $[109,115,119]$. The alkaloids with (R)-apomorphine groups have been clinically used for the treatment of Parkinson's disease [144].

\subsubsection{Acetogenins (ACGs)}

ACGs are molecules characterized by a long aliphatic chain of 35 to 37 carbon atoms with one, two, or three tetrahydrofuran or tetrahydropyran rings in their central region [124]. Annonaceae acetogenins are secondary metabolites with the highest therapeutic interest, due to their cytotoxic capacity on cancer cell lines and their in vivo antitumoral activity [82]. The acetogenins are found in the seeds (A. muricata, A. cherimola "A. diversifolia, A. squamosa, and $A$. reticulata), leaves (A. muricata, $A$. cherimola, and $A$. squamosa), edible pulp (A. muricata 
and A. squamosa), roots (A. muricata), and stem bark (A. muricata, A. cherimola, and $A$. squamosa). Although there are more than five hundred identified acetogenins, annonacin and bullatacin are considered the acetogenins with the highest concentration in Annona species. Moreover, they have excellent cytotoxicity at low doses, but they can be highly toxic if consumed in high concentrations [82,145]. They can be found in the pulp, leaves, seeds, and roots of $A$. muricata $[83,124,125,127,134]$. In the same way, annonacin and bullatacin have been identified in the pulp and stem bark of $A$. squamosa $[135,138]$. In A. macroprophyllata, the principal reported acetogenins from seeds are rolliniastatin-2, laherradurin, and cherimolin-2 [88,89].

\subsection{Biological Activities from Extracts or Isolated Compounds from Annona Species}

There are many biological activities attributed to extracts (aqueous, ethanolic, methanolic, and ethyl acetate) or isolated compounds from Annona species such as anti-angiogenic, analgesic, thrombolytic, anti-ulcer, anti-platelet, vasorelaxant, anti-pyretic, anti-convulsant, anti-depressive, neuroprotective, anxiolytic, antitumoral or cytotoxic, hypoglycemic, and anti-inflammatory [146]. Table 4 shows several studies on the biological effects of extracts or isolated compounds from principal fruit Annonas.

\subsubsection{Cytotoxic Activity}

Cytotoxic activity has been studied in fruit, leaves, root, and stem bark extracts or isolated acetogenins of some Annona species (Table 4). Annonacin at a very low dose (extracted from A. muricata leaves) decreased the induced-tumors on mice [147,148]; moreover, acetogenins have a potent cytotoxicity against many cancer cell lines (DU-145, A-549, MCF-7, HT-29, A-498, PC-3, HL60, HCT, Caco-2, and HepG-2) [88,96,119,137,143,149-152]. The mechanism of action of acetogenins on cancer cell lines or in vivo cancer models is attributed to inhibition mitochondrial complex I (ubiquinone-linked NADH oxidase) to present in cell membranes; the ATP decrease cause apoptosis [123].

Table 4. Cytotoxicity, anti-hyperglycemic, and anti-inflammatory activities of different crude extracts or isolated compounds from different plant parts of some Annona species using different model assays.

\begin{tabular}{|c|c|c|c|c|c|c|}
\hline $\begin{array}{c}\text { Activityl } \\
\text { Annona Species }\end{array}$ & Plant Part & Bioactive & Dose/Concentration & Model Assay & Effect & References \\
\hline \multicolumn{7}{|c|}{ Cytotoxicity } \\
\hline \multirow{4}{*}{ A. muricata } & \multirow[t]{2}{*}{ Leaf } & Annonacin & $85 \mathrm{nM}$ & $\begin{array}{l}\text { Mice induced a skin } \\
\text { tumorogenesis }\end{array}$ & $\begin{array}{l}\text { To reduce the tumor } \\
\text { incidence, tumor } \\
\text { burden, and } \\
\text { tumor volume }\end{array}$ & [147] \\
\hline & & $\begin{array}{l}\text { Acetogenin-rich } \\
\text { fraction }\end{array}$ & $\begin{array}{l}100 \text { and } 200 \mathrm{mg} / \mathrm{kg} \\
\text { for } 7 \text { days }\end{array}$ & $\begin{array}{l}\text { Rats induced benign } \\
\text { prostatic hyperplasia } \\
\text { (PSA) }\end{array}$ & $\begin{array}{c}\text { The fractions } \\
(200 \mathrm{mg} / \mathrm{kg}) \\
\text { significantly reduced } \\
\text { the PSA level }\end{array}$ & [148] \\
\hline & $\begin{array}{l}\text { Root-bark, } \\
\text { fruit, leaf }\end{array}$ & $\begin{array}{l}\text { Ethyl acetate } \\
\text { extract }\end{array}$ & $50 \mu \mathrm{g} / \mathrm{mL}$ & $\begin{array}{c}\text { MCF-10A cell line } \\
\text { (breast) }\end{array}$ & $\begin{array}{l}\text { The fractions had the } \\
\text { highest } \\
\text { anticancer abilities }\end{array}$ & [149] \\
\hline & Stem bark & $\begin{array}{c}\text { Annonacin and } \\
\text { ethyl acetate } \\
\text { extract }\end{array}$ & $\begin{array}{c}\mathrm{IC}_{50} 0.1 \mu \mathrm{M} \text { and } \\
55.501 \mu \mathrm{g} / \mathrm{mL}\end{array}$ & $\begin{array}{l}\text { DU-145 prostate } \\
\text { carcinoma cells }\end{array}$ & $\begin{array}{c}\text { Annonacin and } \\
\text { extract displayed } \\
\text { selective and potent } \\
\text { cytotoxicity }\end{array}$ & {$[150]$} \\
\hline A. cherimola & Seed & $\begin{array}{l}\text { Annomolin, } \\
\text { annocherimolin }\end{array}$ & & $\begin{array}{c}\text { A-549 (lung), MCF-7 } \\
\text { (breast), HT-29 } \\
\text { (colon), A-498 } \\
\text { (kidney), PC-3 } \\
\text { (prostate) and MIA } \\
\text { PaCa-2 (pancreas) } \\
\text { cell lines }\end{array}$ & $\begin{array}{l}\text { Potent cytotoxicity } \\
\text { against all cell lines }\end{array}$ & [137] \\
\hline
\end{tabular}


Table 4. Cont.

\begin{tabular}{|c|c|c|c|c|c|c|}
\hline $\begin{array}{c}\text { Activity/ } \\
\text { Annona Species }\end{array}$ & Plant Part & Bioactive & Dose/Concentration & Model Assay & Effect & References \\
\hline & & Ethanolic extract & $\mathrm{IC}_{50} 23.20 \mu \mathrm{g} / \mathrm{mL}$ & $\begin{array}{l}\text { Colorectal cancer cell } \\
\text { lines: T84, HCT-15, } \\
\text { SW480 and HT-29, } \\
\text { cancer stem cells }\end{array}$ & $\begin{array}{l}\text { Potential cytotoxic } \\
\text { activity on T- } 81 \text { and } \\
\text { HCT- } 15 \text { resistant } \\
\text { cell lines }\end{array}$ & [151] \\
\hline & Leaf & Ethanolic extract & $\mathrm{IC}_{50} 390.20 \mu \mathrm{g} / \mathrm{mL}$ & $\begin{array}{l}\text { Breast cancer cell } \\
\text { lines: MDA-MB-231 } \\
\text { and MCF-7 }\end{array}$ & $\begin{array}{c}\text { Selective } \\
\text { antiproliferative and } \\
\text { pro-apoptotic } \\
\text { activities }\end{array}$ & [152] \\
\hline \multirow{4}{*}{ A. squamosa } & Stem bark & Bullatacin & $\begin{array}{c}\mathrm{IC}_{50} \text { of } \\
2.47 \times 10^{-7} \mu \mathrm{g} / \mathrm{mL}\end{array}$ & $\begin{array}{c}\text { A-549, HT-29, MCF-7, } \\
\text { A-498, PC3 and } \\
\text { PACA-2 cell lines }\end{array}$ & $\begin{array}{l}\text { Selective cytotoxic } \\
\text { activity against } \\
\text { MCF-7 cells }\end{array}$ & [142] \\
\hline & Seed & Acetogenins & $\begin{array}{c}\mathrm{IC}_{50} \text { ranged from } \\
2.2 \times 10^{-1} \text { to } \\
8.3 \times 10^{-3} \mu \mathrm{g} / \mathrm{mL}\end{array}$ & $\begin{array}{l}\text { HeLa, MCF-7, A-549, } \\
\text { Hep-G2, SMMC-7721 } \\
\text { and MKN-45 cell lines }\end{array}$ & $\begin{array}{l}\text { Selective cytotoxic } \\
\text { activity against } \\
\text { MCF-7 and } \\
\text { A-549 cells }\end{array}$ & [141] \\
\hline & Leaf & $\begin{array}{l}\text { Methanolic } \\
\text { extract }\end{array}$ & $\begin{array}{l}\mathrm{IC}_{50} \text { ranged from } 1.1 \\
\text { to } 2.1 \mu \mathrm{g} / \mathrm{mL}\end{array}$ & $\begin{array}{l}\text { Human immortalized } \\
\text { line of T lymphocyte } \\
\text { (Jurkat), MCF-7, } \\
\text { HL60, and HCT-116 }\end{array}$ & $\begin{array}{l}\text { Leaf extract was more } \\
\text { active against MCF-7 } \\
\text { cells, likewise for seed } \\
\text { extract against Jurkat } \\
\text { and HL60 cells }\end{array}$ & [119] \\
\hline & $\begin{array}{l}\text { Seed, peel, } \\
\text { pulp }\end{array}$ & Aqueous extract & $\begin{array}{l}\mathrm{IC}_{50} 7.31 \pm 0.03 \text { and } \\
15.99 \pm 1.25 \mu \mathrm{g} / \mathrm{mL}\end{array}$ & $\begin{array}{l}\text { Cancer cell lines colon } \\
\text { (Caco-2), prostate } \\
\text { (PC3), liver (HepG-2), } \\
\text { and breast (MCF-7) }\end{array}$ & $\begin{array}{l}\text { Seed extracts had the } \\
\text { lowest } \text { IC }_{50} \text { values for } \\
\text { PC-3 and MCF-7 } \\
\text { cancer cell lines }\end{array}$ & [96] \\
\hline \multirow{2}{*}{$\begin{array}{c}\text { A. } \\
\text { macroprophyllata }\end{array}$} & \multirow[t]{2}{*}{ Seed } & Cherimolin-2 & $\begin{array}{l}\mathrm{IC}_{50} \text { of } 0.5 \mu \mathrm{g} / \mathrm{mL} \text { for } \\
\text { SW- } 480 \text { and for HeLa } \\
0.05 \mu \mathrm{g} / \mathrm{mL} \text {. In vivo } \\
\text { doses } 500 \mathrm{mg} / \mathrm{kg} \\
\text { body weight, } 20 \text { days }\end{array}$ & $\begin{array}{c}\text { HeLa and SW-480 cell } \\
\text { line and rats injected } \\
\text { with HeLa }\left(1 \times 10^{6}\right) \\
\text { or SW-480 } \\
\left(5 \times 10^{6}\right) \text { cells }\end{array}$ & $\begin{array}{c}\text { Size reduction of } \\
\text { HeLa tumor }(43 \%), \\
\text { and } 16 \% \text { of SW- } 480 \\
\text { tumor }\end{array}$ & [88] \\
\hline & & Laherradurin & $\begin{array}{c}\mathrm{IC}_{50} \text { of } 0.15 \mu \mathrm{g} / \mathrm{mL} \\
\text { for HeLa and SW- } 480 . \\
\text { In vivo doses } \\
500 \mathrm{mg} / \mathrm{kg} \text { body } \\
\text { weight } 20 \text { days }\end{array}$ & $\begin{array}{c}\text { HeLa and SW-480 cell } \\
\text { line and rats injected } \\
\text { with HeLa }\left(1 \times 10^{6}\right) \\
\text { or SW-480 } \\
\left(5 \times 10^{6}\right) \text { cells }\end{array}$ & $\begin{array}{l}\text { Size reduction of } \\
\text { HeLa tumor }(64 \%) \\
\text { and SW-480 tumor } \\
(60 \%)\end{array}$ & [88] \\
\hline \multicolumn{7}{|c|}{ Hypoglycemic activity } \\
\hline \multirow{2}{*}{ A. muricata } & \multirow{2}{*}{ Leaf } & Aqueous extract & $\begin{array}{l}100 \mathrm{mg} / \mathrm{kg} \text { for } \\
2 \text { weeks }\end{array}$ & $\begin{array}{l}\text { Streptozotocin- } \\
\text { induced } \\
\text { diabetic rats, }\end{array}$ & $\begin{array}{l}\text { Significant reduction } \\
\text { of blood glucose } \\
\text { levels and protective } \\
\text { action on pancreatic } \\
\beta \text {-cells }\end{array}$ & [153] \\
\hline & & Dry extract & $\begin{array}{c}100 \mathrm{mg} / \mathrm{kg} / \text { day for } \\
4 \text { weeks }\end{array}$ & $\begin{array}{l}\text { Streptozotocin- } \\
\text { induced } \\
\text { diabetic rats. }\end{array}$ & $\begin{array}{l}\text { The dry extract } \\
\text { improves behavioral } \\
\text { alterations and } \\
\text { protects testis in } \\
\text { diabetic animals }\end{array}$ & [154] \\
\hline \multirow{3}{*}{ A. cherimola } & Steam bark & Ethanolic extract & $\begin{array}{l}150 \text { and } 300 \mathrm{mg} / \mathrm{kg} \\
\text { for } 2 \text { weeks }\end{array}$ & $\begin{array}{l}\text { Alloxan-induced } \\
\text { diabetic male } \\
\text { albino rats }\end{array}$ & & [155] \\
\hline & \multirow[b]{2}{*}{ Leaf } & Ethanolic extract & $300 \mathrm{mg} / \mathrm{kg}$, one week & $\begin{array}{l}\text { Alloxan-induced type } \\
2 \text { diabetic (AITD) }\end{array}$ & $\begin{array}{c}\text { Attenuated } \\
\text { postprandial } \\
\text { hyperglycemia }\end{array}$ & [156] \\
\hline & & $\begin{array}{l}\text { Aqueous extract } \\
\text { (infusion) } \\
(1.5 \mathrm{~g} / \mathrm{mL})\end{array}$ & $300 \mathrm{mg} / \mathrm{kg}$ & $\begin{array}{l}\text { Streptozocin-induced } \\
\text { diabetic mice }\end{array}$ & $\begin{array}{l}\text { Reduction of the } \\
\text { blood glucose level, } \\
\text { glycated hemoglobin, } \\
\text { cholesterol, and } \\
\text { triacylglycerols }\end{array}$ & [157] \\
\hline
\end{tabular}


Table 4. Cont.

\begin{tabular}{|c|c|c|c|c|c|c|}
\hline $\begin{array}{c}\text { Activity/ } \\
\text { Annona Species }\end{array}$ & Plant Part & Bioactive & Dose/Concentration & Model Assay & Effect & References \\
\hline \multirow{4}{*}{ A. squamosa } & \multirow[b]{2}{*}{ Leaf } & Aqueous extract & $\begin{array}{c}300 \mathrm{mg} / \mathrm{kg} \text { body } \\
\text { weight }\end{array}$ & $\begin{array}{l}\text { Streptozotocin- } \\
\text { induced } \\
\text { Wistar rats } \\
\end{array}$ & $\begin{array}{c}\text { Decreased glucose } \\
\text { and increased insulin } \\
\text { sensitivity }\end{array}$ & [158] \\
\hline & & Ethanolic extract & $\begin{array}{c}100 \mathrm{mg} / \mathrm{kg} \text { body } \\
\text { weight }\end{array}$ & $\begin{array}{l}\text { Streptozotocin- } \\
\text { induced } \\
\text { rats }\end{array}$ & $\begin{array}{l}\text { Glycemia decreased } \\
\text { as well as glycated } \\
\text { hemoglobin, } \\
\text { creatinine, and urea }\end{array}$ & [159] \\
\hline & Root & Aqueous extract & $\begin{array}{c}500 \mathrm{mg} / \mathrm{kg} \text { body } \\
\text { weight }\end{array}$ & $\begin{array}{l}\text { Streptozotocin- } \\
\text { induced } \\
\text { hyperglycemic rats }\end{array}$ & $\begin{array}{c}\text { A significant } \\
\text { reduction of glycemia, } \\
6 \mathrm{~h} \text { after oral } \\
\text { administration }\end{array}$ & [160] \\
\hline & $\begin{array}{l}\text { Leaf and } \\
\text { peel }\end{array}$ & Aqueous extract & $\begin{array}{c}250 \mathrm{mg} / \mathrm{kg} \text { body } \\
\text { weight }\end{array}$ & $\begin{array}{l}\text { Streptozotocin- } \\
\text { induced diabetic } \\
\text { rats }\end{array}$ & $\begin{array}{l}\text { Improvement of the } \\
\text { glycemia and lipid } \\
\text { profile }\end{array}$ & [161] \\
\hline \multirow[b]{2}{*}{$\begin{array}{c}\text { A. } \\
\text { macroprophyllata }\end{array}$} & Leaf & Ethanolic extract & $\begin{array}{c}200 \mathrm{mg} / \mathrm{kg} \text { body } \\
\text { weight }\end{array}$ & $\begin{array}{l}\text { Alloxan-induced } \\
\text { diabetic Balb-c mice }\end{array}$ & $\begin{array}{l}\text { A significant decrease } \\
\text { in postprandial } \\
\text { hyperglycemia }\end{array}$ & [162] \\
\hline & & Aqueous extract & $\begin{array}{c}300 \mathrm{mg} / \mathrm{kg} \text { body } \\
\text { weight }\end{array}$ & Healthy rats & $\begin{array}{c}\text { Reduction of } \\
\text { glycemia at a } \\
\text { dose-dependent } \\
\text { manner }\end{array}$ & [163] \\
\hline \multicolumn{7}{|c|}{ Anti-inflammatory activity } \\
\hline \multirow{3}{*}{ A. muricata } & Fruit & Aqueous extract & $\begin{array}{l}50,100 \text { and } \\
200 \mathrm{mg} / \mathrm{kg}\end{array}$ & $\begin{array}{l}\text { Carrageenan-induced } \\
\text { paw edema rats and } \\
\text { xylene-induced-ear } \\
\text { edema rats }\end{array}$ & $\begin{array}{c}\text { Significant } \\
\text { anti-inflammatory } \\
\text { activity on paw } \\
\text { edema and ear edema }\end{array}$ & [164] \\
\hline & \multirow{2}{*}{ Leaf } & Aqueous extract & $\begin{array}{c}100 \mathrm{mg} / \mathrm{kg} \text { body } \\
\text { weight }\end{array}$ & $\begin{array}{l}\text { Female Balb/c albino } \\
\text { mice injected with } \\
\text { Escherichia fergusonii }\end{array}$ & $\begin{array}{l}\text { The extract } \\
\text { minimized the } \\
\text { inflammation by } \\
\text { decreasing the } \\
\text { expression levels of } \\
\text { IL-1 } \beta \text { and TNF- } \alpha\end{array}$ & [165] \\
\hline & & Ethanolic extract & $\begin{array}{c}100,200, \text { and } \\
400 \mathrm{mg} / \mathrm{kg} \text { orally for } \\
7 \text { days }\end{array}$ & $\begin{array}{l}\text { Rectoanal tissue from } \\
\text { Swiss mice }\end{array}$ & $\begin{array}{l}\text { All three doses show } \\
\text { significant } \\
\text { anti-inflammatory } \\
\text { effects on } \\
\text { hemorrhoidal tissue }\end{array}$ & [166] \\
\hline A. cherimola & Leaf & Ethanolic extracts & $100 \mathrm{mg} / \mathrm{kg}$ & $\begin{array}{l}\text { Rats (Leukocyte } \\
\text { migration to the } \\
\text { peritoneal cavity and } \\
\text { Subcutaneous air } \\
\text { pouch test) }\end{array}$ & $\begin{array}{l}\text { The inhibitory effect } \\
\text { of the ethanolic } \\
\text { extract on leukocytes } \\
\text { migration was } 63.8 \\
\text { and } 73.16 \%\end{array}$ & [167] \\
\hline \multirow{3}{*}{ A. squamosa } & Root & Ethanolic extract & $\begin{array}{l}400 \mathrm{mg} / \mathrm{kg} \text { body } \\
\text { weight }\end{array}$ & $\begin{array}{l}\text { Carrageenan-induced } \\
\text { paw edema rats }\end{array}$ & $\begin{array}{l}\text { Inhibition (54\%) of } \\
\text { edema inflammation } \\
\text { at } 400 \mathrm{mg} / \mathrm{kg}\end{array}$ & [168] \\
\hline & \multirow[t]{2}{*}{ Stem bark } & $\begin{array}{l}\text { Caryophyllene } \\
\text { oxide }\end{array}$ & $\begin{array}{c}25 \mathrm{mg} / \mathrm{kg} \text { body } \\
\text { weight }\end{array}$ & $\begin{array}{l}\text { Acetic acid-induced } \\
\text { Swiss albino rats }\end{array}$ & $\begin{array}{c}\text { Inhibition of } \\
\text { inflammation }(75 \%) \\
\text { at } 25 \mathrm{mg} / \mathrm{kg}\end{array}$ & [169] \\
\hline & & $\begin{array}{l}\text { 18-acetoxyent- } \\
\text { kaur-16-ene }\end{array}$ & $\begin{array}{l}25 \mathrm{mg} / \mathrm{kg} \text { body } \\
\text { weight }\end{array}$ & $\begin{array}{c}\text { Acetic } \\
\text { acid-induced rats }\end{array}$ & $\begin{array}{c}\text { Inhibition of } \\
\text { inflammation } 62 \%) \text { at } \\
25 \mathrm{mg} / \mathrm{kg}\end{array}$ & [170] \\
\hline
\end{tabular}

\subsubsection{Anti-Hyperglycemic Activity}

Various studies on anti-hyperglycemic effects are reported for Annona species. The antihyperglycemic activity of $A$. squamosa leaves, roots, and peel extracts have been evaluated in hyperglycemic or diabetic models [153-163]. Table 4 shows that leaves have been studied more than other tissues. The glucose reduction in blood by Annona extracts is related to the presence of polyphenols [156]. 


\subsubsection{Anti-Inflammatory}

Anti-inflammatory activity has been explored in fruit, leaves, root, and stem bark extracts of some Annona species [164-170], as shown in Table 4. A. cherimola leaf extract exhibited a potent in vivo anti-inflammatory activity on induced-inflammation rats [167]. The anti-inflammatory activity of Annona extracts is related to the content of some phenolic compounds, alkaloids, kaurenes, or sesquiterpenes in the extracts $[169,170]$.

\subsection{Technologies for Postharvest Handling}

The fruit belonging to the genus Annona are climacteric, with respiration rates of up to $350 \mathrm{~mL}$ of $\mathrm{CO}_{2} \mathrm{~kg}^{-1} \mathrm{~h}^{-1}$, in addition to having ethylene production values of 46.2 and $68.5 \mu \mathrm{L} \mathrm{kg}^{-1} \mathrm{~h}^{-1}$ at a storage temperature of 25 to $30{ }^{\circ} \mathrm{C}$ [171]. Due to the above, the production capacity of Annona is wasted, since the production of these fruit in the country presents various management problems [172]. Moreover, a $60 \%$ post-harvest loss has been reported, which causes the producer to choose to move their fruit in transport with high cost (air) and use expensive post-harvest technologies to delay the ripening of the fruit, mainly refrigeration $\left(13^{\circ} \mathrm{C}\right)$ [173].

During the handling of the fruit, it is essential to preserve the physical, chemical, and organoleptic characteristics that are ideal for the consumer [174], which is why the essential result is to carry out an adequate postharvest handling. However, there are no postharvest technologies registered or issued by organizations for the handling of fruit belonging to the genus Annona [175]. The considerations for the post-harvest handling of soursop (Annona muricata L.) that are applied are those described by Morton, where it is mentioned that the fruit needs to be kept under conditions of room temperature and relative humidity of 85 and $90 \%$. In addition, it is necessary to be cautious when handling the fruit, since they are susceptible to physical, chemical, and microbiological damage [176]. The common operations in the post-harvest handling of soursop are harvesting, selection, cleaning of organic residues, classification, antifungal treatment, weighing, pre-cooling $\left(12\right.$ to $\left.15^{\circ} \mathrm{C}\right)$, drying of residual humidity, post-harvest treatment (waxing), storage, and transport [177].

As mentioned above, high rates of respiration and ethylene production play an important role in the postharvest handling of the genus Annona. Ethylene is responsible for regulating the maturation and senescence of various agricultural products [178]; therefore, it is responsible for the fruit to acquire optimal organoleptic characteristics for consumption, as well as for the senescence of the fruit [179]. Eventually, having identified the mechanisms of action of ethylene on the fruit, it has been possible to develop technologies and procedures to control the different negative effects that this hormone can cause (Table 1). In research carried out by Wijesinghe et al., the effect of 1-methylcyclopropene (1-MCP) at $0.3,0.6$, and $0.9 \mu \mathrm{L} / \mathrm{L}$ was evaluated in four different Annona fruit: A. atemoya, A. reticulata, A. muricata, and A. squamosa. As response variables, the days of storage, physiological weight loss (PWL), firmness, color, total soluble solids (TSS), and titratable acidity (TA) were measured. At the end of the investigation, it was concluded that the application of $1-\mathrm{MCP}$ increased the shelf life to three extra days for all the fruit compared to the control fruit without treatment. The $0.6 \mu \mathrm{L} / \mathrm{L}$ concentration reduced $\mathrm{PWL}$, the enzyme activity responsible for the loss of firmness and control of chlorophyll degradation in addition to regular TSS and TA [180].

Other technologies used to extend the shelf life of perishable fruit are coatings or so-called "waxes". Montalvo-González et al. [181] used candelilla and beeswax emulsions as postharvest technology applied to soursop fruit (A. muricata). Respiration rate, ethylene production, $\mathrm{PWL}$, firmness, TSS, TA, $\mathrm{pH}$, and color $\left({ }^{\circ}\right.$ hue) were considered as response variables. The authors describe a favorable effect on respiration rate, since it decreased in the fruit treated with wax and managed to delay the appearance of the climacteric peak one day after the control fruit. A similar effect was found in the rate of ethylene production since the levels of the treated fruit were lower compared to the control fruit. Finally, for TSS, TA, $\mathrm{pH}$, and color, a moderating effect was noted in the generation of these parameters since the metabolic processes of the fruit were not inhibited or modified [181]. 
By showing an interesting effect on the genus Annona, 1-MCP and waxes were studied in combination due to their high performance and potential applications.

The research of Tovar et al. [173] was based on applying to soursop fruit $1000 \mathrm{~nL} / \mathrm{L}$ of 1-MCP in combination with three different wax formulations: first, carnauba wax type III Emulwax 3060; second, carnauba wax type III, and food-grade silicone oils Emulwax 3061; and third, refined candelilla wax Emulwax 3070 (all formulations were applied after $1-\mathrm{MCP})$. The determinations that were used as response variables were PWL, TSS, TA, $\mathrm{pH}$, color $\left({ }^{\circ}\right.$ hue), and firmness. In general, the combination of 1-MCP and the three types of coatings managed to lengthen the shelf life of the fruit by an extra six days, as compared to the control (18 days in total). In the PWL analyses, it was discovered that the combination of these technologies managed to significantly reduce this parameter; however, no difference was found between the treatments. Regarding the content of TSS, TA and $\mathrm{pH}$ were observed a major delay in the normal evolution of the variables. The values of Hue for the control fruit and combination 1 and 2 did not show significant differences; however, for combination 3, a decrease in color development was noted. The author attributed this phenomenon to the synergistic effect of formulations 1 and 3. Finally, in the firmness data, was reported significant differences between the treated and control fruit, but no differences were found between treatments [173]. Table 5 shows some technologies to extend the shelf life of Annona fruits.

Table 5. Postharvest technologies or treatments to extend the shelf life of fruit of the genus Annona.

\begin{tabular}{|c|c|c|c|}
\hline Technology/Treatment & Genus Annona & Effect & References \\
\hline $\begin{array}{l}\text { Aqueous extract of coconut mesocarp } \\
+ \text { commercial chitosan }\end{array}$ & Annona muricata $\mathrm{L}$. & Control of Rhizopus stolonifera & [182] \\
\hline Electrolyzed solution with neutral $\mathrm{pH}$ & Annona muricata $\mathrm{L}$. & Sporicidal activity & [183] \\
\hline $\begin{array}{c}\text { Hydrothermal, fungicidal, and wax } \\
\text { treatment }\end{array}$ & Annona muricata $\mathrm{L}$. & Browning control & [184] \\
\hline Edible mucilage coating & Annona muricata $\mathrm{L}$. & $\begin{array}{l}\text { Preservation of the physicochemical } \\
\text { characteristics and extending the shelf } \\
\text { life ( } 2 \text { extra days) }\end{array}$ & [185] \\
\hline 1-Methylcyclopropene & Annona muricata $\mathrm{L}$. & $\begin{array}{l}\text { Preservation of physicochemical } \\
\text { characteristics }\end{array}$ & [186] \\
\hline Chitosan coating & Annona muricata $\mathrm{L}$. & Anthracnose control & [187] \\
\hline Chitosan coating & Annona muricata $\mathrm{L}$. & $\begin{array}{l}\text { Control of physicochemical, } \\
\text { microbiological, and sensory } \\
\text { characteristics }\end{array}$ & [188] \\
\hline Cold storage & Annona muricata $\mathrm{L}$. & $\begin{array}{l}\text { Increase the days of shelf life of the fruit } \\
\qquad(2 \text { extra days })\end{array}$ & [189] \\
\hline Bacillus atrophaeus strain B5 & Annona muricata $\mathrm{L}$. & Biocontrol of postharvest anthracnose & [190] \\
\hline
\end{tabular}

\section{Perspectives}

Since the agronomic knowledge of these fruit species of Annona in Mexico is still limited, it is necessary to exploit the conditions of the center of origin of most of these and the optimal climatic conditions for their cultivation. Therefore, the Red Nacional de Annonaceae should initially focus its activities on the generation of knowledge of best agronomic practices that allow for obtaining the highest yields and the least environmental impact of these crops to achieve their sustainable development. There is a lack of selected varieties and characterization of the great gene pool that is still found in natural conditions, backyard, or underutilized on the verges of harvesting roads or grazing areas. It is necessary to carry out an exhaustive search, characterize and begin an improvement program with the most outstanding productive genotypes or with some characteristics of interest. The potential for commercial use of these species is underutilized, given the great acceptance of the fruits for fresh consumption, and obtaining products and applications in areas such as medicine or therapeutic treatments. Currently, it is still important to look for extraction alternatives to increase the yield and demonstrate the distribution and contents 
of compounds in the different Annona species. Although these species are rich in secondary metabolites, it is not fully clear if they can be a source of nutraceutical metabolites for the pharmaceutical industry. According to the review, various extracts of $A$. muricata, $A$. cherimola, A. squamosa, $A$. macroprophyllata, and $A$. reticulata species contain metabolites capable of exerting different biological activities that are beneficial to health; however, further studies on the precise mechanisms of action and expanding the knowledge of Annona species less investigated such as $A$. reticulata are required.

Author Contributions: L.M.H.F.: Conceptualization, investigation, writing draft preparation, writing-review and editing. E.M.G.: Conceptualization, investigation and writing draft preparation. M.d.L.G.M.: investigation and writing draft preparation. L.M.A.E.: investigation and writing draft preparation. Y.N.G.: investigation and writing draft preparation. Z.V.: investigation and writing draft preparation. S.G.T.: investigation and writing draft preparation. J.J.V.M.: writing-review, editing and supervision. D.A.M.F.: Investigation. All authors have read and agreed to the published version of the manuscript.

Funding: This research received no external funding.

Institutional Review Board Statement: Not applicable.

Informed Consent Statement: Not applicable.

Data Availability Statement: The information presented here is the authors' revision. The tables and figures are self-made based on the information and review carried out.

Acknowledgments: We thank the Red Nacional de Anonáceas and Sociedad Mexicana de Anonáceas for sharing information about the current state of Annonaceae in Mexico.

Conflicts of Interest: The authors declare no conflict of interest.

\section{References}

1. Prasad, M.; Singh, H.; Singh, S.K. Early Eocene Annona fossils from Vastan Lignite Mine, Surat district, Gujarat, India: Age, origin and palaeogeographic significance. Curr. Sci. 2014, 107, 1730-1735.

2. Chatrou, L.W. The Annonaceae and the Annonaceae Project: A brief overview of the state of affairs. Acta Hortic. 1999, 497, 43-49. [CrossRef]

3. GBIF. Annonaceae in GBIF Secretariat (2021). GBIF Backbone Taxonomy. Checklist Dataset. Available online: https://doi.org/10 $.15468 / 39$ omei (accessed on 15 September 2021).

4. WCVP. World Checklist of Vascular Plants, Version 2.0. Facilitated by the Royal Botanic Gardens, Kew. Available online: http:/ / wcvp.science.kew.org/ (accessed on 15 September 2021).

5. CONABIO. Catálogo de Autoridades Taxonómicas de Especies de Flora y Fauna con Distribución en México. Base de Datos SNIB-CONABIO, México. Available online: https:/ /www.snib.mx/taxonomia/descarga/ (accessed on 15 September 2021).

6. Larranaga, N.; Albertazzi, F.J.; Hormaza, J.I. Phylogenetics of Annona cherimola (Annonaceae) and some of its closest relatives. J. Syst. Evol. 2018, 57, 211-221. [CrossRef]

7. Li, P.; Thomas, D.C.; Saunders, R.M.K. Historical biogeography and ecological niche modelling of the Asimina-Disepalum clade (Annonaceae): Role of ecological differentiation in Neotropical-Asian disjunctions and diversification in Asia. BMC. Evol. Biol. 2017, 17, 188. [CrossRef]

8. MacCaughey, V. The Genus Annona in The Hawaiian Islands. Torreya 1917, 17, 69-77. Available online: http:/ /www.jstor.org/ stable/ 40595803 (accessed on 10 September 2021).

9. Laca, M.d.L.; Ramón, L. Las plantas Americanas en la obra de charles de L'écluse: Primeras citas en las cartas de juan castañeda. Anales Jard. Bot. Madrid 1999, 57, 97-107.

10. Colunga, G.M.P.; Zizumbo, V.D. Domestication of plants in maya lowlands. Econ. Bot. 2004, 58, 101-110. [CrossRef]

11. Yadav, V.; Singh, A.K.; Mishra, D.S.; Singh, S. Ramphal (Annona reticulate). In Minor Fruits: Nutraceutical Importance and Cultivation; Ghosh, S.N., Ed.; Jaya Publishing House: Delhi, India, 2017; pp. 829-836.

12. SIAP. Servicio de Información Agroalimentaria y Pesquera. Available online: https://nube.siap.gob.mx/cierreagricola/ (accessed on 16 September 2021).

13. Lemos, P.d.E.E. Panorama de las anonas cultivadas en Brasil: Saramuyo, guanabana y atemoya. In Anonáceas: Plantas Antiguas, Estudios Recientes; González, E.A.R., Luna, C.L.M., Gutierrez, J.J., Schlie, G.M.A., Vidal, L.D.G., Eds.; Colección Jaguar Unicach: Chiapas, Mexico, 2011; pp. 21-36.

14. Safford, W.E. Annona diversifolia, a custard-apple of Aztecs. J. Wash. Acad. Sci. 1912, 2, 118-1125.

15. Safford, W.E. The genus Annona: The derivation of its name and its taxonomic subdivisions. J. Wash. Acad. Sci. 1911, 1, 118-120. 
16. Ballesteros, P.G.; Zavala, H.F.; Vergara, H.R.; Cortés, S.J. Conservación, Mejoramiento y Fomento de la Ilama (Annona diversifolia Saff.) en la Tierra Caliente del Balsas. Foro Estud. Sobre Guerr. 2015, 1, 567-572.

17. Gonzalez, E.A.R.; De la Cruz, C.I.; Castro, M.M.; Riley, S.C.A. Phenological strategies of Annona species from the tropical deciduous forest of Chiapas, México. Bot. Sci. 2016, 94, 531-541. [CrossRef]

18. Cruz, C.J.G. El Cultivo de la Guanabana y su Manejo Agronomico; Universidad Autonoma Metropolitana: Ciudad de México, Mexico, 1988; Volume 19, 40p.

19. Lemos, P.E.E.; Blake, J. Micropropagation of juvenile and adult Annona squamosa. Plant Cell Tiss. Organ. Cult. 1996, 46, 77-79. [CrossRef]

20. Ferreira, N.F.; De Lima, G.S.; Gheyi, H.R.; Da Sá, S.F.V.; Dias, A.S.; Pinheiro, F.W.A. Photosynthetic efficiency and production of Annona squamosa L. under salt stress and fertilization with NPK. Rev. Bras. Eng. Agríc. Ambient. 2021, 25, 446-452. [CrossRef]

21. Bánki, O.; Roskov, Y.; Vandepitte, L.; DeWalt, R.E.; Remsen, D.; Schalk, P.; Orrell, T.; Keping, M.; Miller, J.; Aalbu, R.; et al. Catalogue of Life Checklist (Version 2021-08-25). Catalogue of Life. 2021. Available online: https://doi.org/10.48580/d4sg (accessed on 20 September 2021).

22. Hernández, F.L.M.; Luna, E.G. Uso potencial de anonas, chirimoyas y guanábanas (Genero Annona). In La Biodiversidad en Nayarit. Estudio de Estado; CONABIO: Ciudad de México, Mexico, 2021; Volume II, pp. 305-308.

23. Hernández, F.L.M.; Andrés, A.J.; Espíndola, B.M.d.C.; Castañeda, V.A.; Ballesteros, P.G.; Vera, S.K.S. Recursos genéticos de anonáceas (Annonaceae) en México: Situación actual y perspectivas. Agro Produc. 2016, 9, 3-8.

24. González, E.A.R.; Luna, C.L.M.; Gutiérrez, J.J.; Schlie, G.M.A.; Vidal, L.D.G. (Eds.) Anonáceas: Plantas Antiguas, Estudios Reciente. Parte III; Universidad de Ciencias y Artes de Chiapas: Tuxtla Gutierrez, Chiapas, Mexico, 2011; pp. 15-541.

25. Andres, A.J. Situación actual de las investigaciones de las anonáceas en México. In Anonáceas. Plantas Antiguas. Estudios Recientes. Parte 2; Vidal, L.E., Vidal, M.N.A., y Vidal, H.L., Eds.; Universidad Autónoma Chapingo: Texcoco, Edo de México, Mexico, 2015; pp. 27-39.

26. DOF. Acuerdo por el que se crea el Comité Sectorial de Recursos Genéticos para la Alimentación y la Agricultura. 2020, delivered on 16 July 2020. Available online: https:/ / www.fao.org/plant-treaty/herramientas/toolbox-for-sustainable-use/details/es/c/ 1370735/ (accessed on 30 November 2021).

27. SNICS. Available online: https:// datastudio.google.com/reporting/5b7206ba-e190-48fe-9696-73523bfccf58/page/itBWB (accessed on 1 October 2021).

28. Hernández, F.L.M.; Gómez, J.R.; Andrés, A.J. Importancia, Plagas Insectiles y Enfermedades Fungosas del Cultivo del Guanábano; Libro Técnico Núm. 1; Instituto Nacional de Investigaciones Forestales, Agrícolas y Pecuarias: Nayarit, Mexico, 2013; 87p.

29. Cruz, P.E. Cultivo de Anona; Boletín Técnico 2, Centro Nacional de Tecnología Agropecuaria y Forestal: Ciudad Arce La Libertad, El Salvador, 1988; 20p.

30. Cruz, C.J.G.; Torres, L.P.A.; Delgado, M.J.C.; Domínguez, M.V.M.; Pérez, D.; Franco, M.O. El Guanábano: Agronomía y Usos de Frutales Tropicales; Universidad Autónoma Chapingo: Texcoco, Estado de México, Mexico, 2002; 177p.

31. Hernández, F.L.M.; Bautista, M.N.; Carrillo, S.J.L.; Sánchez, A.H.; Urías, L.M.A.; Salas, A.M.D. Control del barrenador de las semillas, Bephratelloides cubensis Ashmead (Hymenoptera: Eurytomidae) en guanábana, Annona muricata L. (Annonales: Annonaceae). Acta Zool. Mex. 2008, 24, 199-206. [CrossRef]

32. Hernández, F.L.M.; Nolásco, G.Y.; Cruz, G.E.J. Selección y Caracterización de Guanábana y Recomendaciones para su Manejo Agronómico; Folleto Técnico Núm. 34; Instituto Nacional de Investigaciones Forestales, Agrícolas y Pecuarias: Ciudad de México, Mexico, 2017; 64p.

33. Hernández, F.L.M.; Urías, L.M.A.; Baustista, M.N. Biología y hábitos del barrenador de la semilla bephratelloides cubensis ashmead (Hymenoptera: Eurytomidae). Neotrop. Entomol. 2010, 39, 527-534. [CrossRef] [PubMed]

34. Pinzón, G.J.M.; Hernández, F.L.M.; Luna, E.G.; Isiordia, A.N.; Ortíz, C.M. Biología y hábitos de gonodonta pyrgo cramer en Annona muricata. Southwest Entomol. 2016, 41, 251-258. [CrossRef]

35. Hernández, F.L.M.; Nolasco, G.Y.; Orozco, S.M.; Montalvo, G.E. Toxicidad de insecticidas contra Optatus palmaris pascoe en guanábana. Rev. Mexicana Cienc. Agric. 2021, 12, 49-60. [CrossRef]

36. Hernández, F.L.M.; Gómez, J.R.; Orozco, S.M. El Barrenador de las Semillas Bephratelloides cubensis y su Manejo en el Cultivo de Guanábana; Libro Científico Núm. 1; Instituto Nacional de Investigaciones Forestales, Agrícolas y Pecuarias: Ciudad de México, Mexico, 2014; 73p.

37. Nolasco, G.Y.; Hernández, F.L.M.; Montalvo, G.E. Caracterización morfológica y fisicoquímica de accesiones de guanábana seleccionadas en Nayarit. Rev. Mexicana Cienc. Agric. 2019, 10, 223-237.

38. Jiménez, Z.J.O.; Balois, M.R.; Alia, T.I.; Juárez, L.P.; Sumaya, M.M.T.; Bello, L.J.E. Caracterización de frutos de guanábana (Annona muricata L.) en Tepic, Nayarit, México. Rev. Mexicana Cienc. Agric. 2016, 7, 1261-1270.

39. Terán, E.B.; Alia, T.I.; Balois, M.R.; Juárez, L.P.; López, G.G.A.; Pérez, A.; Núñez, C.C.A. Caracterización física, química y morfológica de frutos de guanábana (Annona muricata L.). Agrociencia 2019, 53, 1013-1027.

40. Rodríguez, N.J.R.; Campos, R.E.; Andrés, A.J.; Alia, T.I.; Ortega, A.S.A.; Peña, C.V.; Madera, S.T.J.; Núñez, C.C.A. Distribution, eco-climatic characterisation, and potential growing regions of Annona cherimola Mill. (Annonaceae) in Mexico. Ethnobio. Conserva. 2021, 10, 1-17.

41. Andrés, A.J.; Segura, L.S. Conservación y uso de los recursos genéticos de Annonaceae en México. Rev. Bras. Frutic. 2014, 36, $118-124$. 
42. Domínguez, P.J.; Castañeda, V.A. Guía Técnica Para la Producción de Chirimoya; Fundación Salvador Sánchez Colín CICTAMEX, S.C.: Coatepec de Harinas, Estado de México, Mexico, 2002; 27p.

43. Andrés, A.J. El cultivo de la Chirimoya (Annona cherimola Mill.) en el Estado de Michoacán; Universidad Autónoma Chapingo: Texcoco, Estado de México, Mexico, 1996; 62p.

44. Apolonio, I.; Castañeda, V.A.; Franco, O.; Morales, E.J.; González, A. Influencia de la fuente de polen y su efectividad en la calidad de frutos de chirimoya (Annona cherimola Mill.). Agron. Costarric. 2015, 39, 61-69. [CrossRef]

45. Andrés, A.J.; Nieto, A.R.; Barrientes, P.A.F.; Martínez, D.M.T.; González, A.F.; Segura, L.S.D.; Cruz, C.J.G.; Gallegos, V.C. Varición morfológica de la hoja de chirimoyo. Rev. Chapingo Ser. Hortic. 2004, 10, 103-110. [CrossRef]

46. Espindola, B.M.C.; Flores, P.I.R. Colección de Trabajo de Chirimoya (Annona cherimola Mill.); Fundación Salvador Sánchez Colín CICTAMEX, S.C.: Coatepec de Harinas, Estado de México, México, 2021; Available online: https://www.gob.mx/cms/uploads / attachment/file/232184/Coleccion_de_trabajo_de_chirimoya_annona_cherimola_Mill_.pdf (accessed on 29 October 2021).

47. Tovar, S.A.; Hernández, M.M.; Cristobal, A.J.; Romero, H.R.; Mora, A.G. Escala logarítmica de severidad de la mancha negra (Colletotrichum gloesporioides Penz.) en chirimoya (Annona cherimola Mill.). Rev. Mex. Fitopatol. 2002, 20, $103-109$.

48. Villanueva, A.R.; Yáñez, M.M.J.; Hernández, A.A.M. Especies de Colletotrichum en chirimoya (Annona cherimola Mill.). Agrociencia 2008, 42, 689-701.

49. Villanueva, A.R.; Cárdenas, S.E.; Hernández, A.A.M.; Mora, A.A.; Téliz, O.D. Patogénesis de la antracnosis (Colletrotrichum fragariae) en frutos de chirimoya. Agrociencia 2006, 40, 773-782.

50. García, N.E.S.; Castañeda, V.A.; Franco, M.O.; Sánchez, P.J.R.; Vaca, P.R.; Hernández, F.L.M. Control de Talponia batesi Henrich (Lepidoptera: Tortricidae) mediante embolsado de frutos en chirimoya (Annona cherimola Mill.). Bol. Mus. Entomol. Univ. Val. 2016, 17, 1-7.

51. Perfectti, F.L.P. Genetic diversity in a worldwide collection of cherimoya cultivars. Genet. Resour. Crop. Evol. 2005, 52, 959-966. [CrossRef]

52. González, M.; Hueso, F.J.J.; Cuevas, A.J. Foliar application of urea advances bud break, bloom and harvest in cherimoya (Annona cherimola Mill.). Acta Hort. 2013, 975, 269-274. [CrossRef]

53. González, M.; Cuevas, J. Flowering pattern and fruitful capacity of ‘Fino de Jete' cherimoya shoots. Acta Hort. 2013, 975, $263-267$. [CrossRef]

54. Pinillos, V.; Peinado, S.; González, M. Fruit development and maturation phenology of ‘Fino de Jete' Cherimoya. Acta Hort. 2013, 975, 335-342. [CrossRef]

55. Alique, R.; Zamorano, J.P.; Calvo, M.L.; Merodio, C.; José, L. Tolerance of cherimoya (Annona muricata Mill.) to cold storage. J. Amer. Soc. Hort. Sci. 1994, 119, 524-528. [CrossRef]

56. Ballesteros, P.G.; Rodríguez, P.L.A.; De la Paz, R.J.; Urieta, P.M.; Zavala, H.F.; Ballesteros, N.N.; Martínez, S.J.P. Diversidad en las Ilamas (Annona diversifolia Saff.) de la Tierra Caliente del Balsas; Garabato Editorial: Guerrero, Mexico, 2010; 60p.

57. Julian, L.A.P.; Santos, S.N.F.; Valadez, B.R.; Sánchez, G.B.S.; Salas, C.R. Chemical composition, color and antioxidant activity of three varieties of Annona diversifolia Safford fruits. Ind. Crops Prod. 2011, 34, 1262-1268. [CrossRef]

58. George, A.P.; Nissen, R.L. Annonaceous fruits. In Encyclopedia of Food Sciences and Nutrition; Caballero, B., Ed.; Elsevier: Amsterdam, The Netherlands, 2003; pp. 239-243.

59. León, J. Botánica de los Cultivos Tropicales; Servicio Editorial IICA: Turrialba, Costa Rica, 1987; 445p.

60. Chavan, S.S.; Shamkumar, P.B.; Damale, M.G.; Pawar, D.P. A comprehensive review on Annona reticulata. Int. J. Pharm. Sci. Res. 2013, 5, 45-50.

61. Shivanna, L.M.; Urooj, A. Apoptotic effects of Annona reticulata leaves extract in HT-29 cell lines. Asian J. Biol. Sci. 2019, 12, 820-831. [CrossRef]

62. Pham, V.T.; Quoc, T.L.; Tuan, A.L.; Quoc, H.V.; Viet, D.H. Phytochemical constituents of Annona reticulata and their cytotoxic activity. Lett. Org. Chem. 2020, 17, 206-210. [CrossRef]

63. Subba, B.; Aryal, P. Study of biological activity and chemical constituent of Annona reticulata. J. Inst. Sci. Technol. 2016, 21, 157-163. [CrossRef]

64. Chang, F.R.; Wu, Y.C.; Duh, C.Y.; Wang, S.K. Studies on the acetogenins of Formosan annonaceous plants. II. Cytotoxic acetogenins from Annona reticulata. J. Nat. Prod. 1993, 56, 1688-1694. [CrossRef]

65. Bioversity International. Available online: http://nwfdb.bioversityinternational.org/detail/?uid_fruit=106 (accessed on 4 November 2021).

66. Ma, C.; Chen, Y.; Chen, J.; Li, X.; Chen, Y. A review on Annona squamosa L.: Phytochemicals and biological activities. Am. J. Chin. Med. 2017, 45, 933-964. [CrossRef]

67. Cala, C.L.; Jardines, C.D.; González, F.R.; Barroso, B.A.; Sánchez, H.M.E.; Cruz, V.H. Estudio farmacognóstico preliminar de la especie Annona squamosa L. Rev. Cubana Plant Med. 2018, 23, 12.

68. Victoria, A.M.C.; Morón, R.F.; Morejón, R.Z.; Martínez, G.M.J.; López, B.M. Tamizaje fitoquímico, actividad antiinflamatoria y toxicidad aguda de extractos de hojas de Annona squamosa L. Rev. Cubana Plant Med. 2006, 11, 12.

69. Cituk, C.D.E. Manual de Propagación y Producción de Saramuyo (Annona squamosa L.); Tecnológico Nacional de México: Yucatán, Mexico, 2021; 38p. 
70. Moreno, H.C.L.; Sáyago, A.S.G.; García, G.H.S.; Mata, M.D.O.; Montalvo, G.E. Effect of the application of 1-methylcyclopropene and wax emulsions on proximate analysis and some antioxidants of soursop (Annona muricata L.). Sci. World J. 2014, $2014,896853$. [CrossRef]

71. Badrie, N.; Schauss, A.G. Soursop (Annona muricata L.): Composition, nutritional value, medicinal uses, and toxicology. Bioact. Foods Promot. Health 2010, 621-643. [CrossRef]

72. Albuquerque, T.G.; Santos, F.; Sanches, S.A.; Beatriz, O.M.; Bento, A.C.; Costa, H.S. Nutritional and phytochemical composition of Annona cherimola Mill. fruits and by-products: Potential health benefits. Food Chem. 2014, 193, 187-195. [CrossRef]

73. Gentile, C.; Mannino, G.; Palazzolo, E.; Gianguzzi, G.; Perrone, A.; Serio, G.; Farina, V. Pomological, sensorial, nutritional and nutraceutical profile of seven cultivars of Cherimoya (Annona cherimola Mill). Foods 2021, 10, 21. [CrossRef] [PubMed]

74. USDA. Agricultural Research Service. Cherimoya, Raw. Available online: https://ndb.nal.usda.gov/fdc-app.html\#/fooddetails /173953/nutrients (accessed on 17 October 2021).

75. Bhardwaj, R.; Pareek, S.; Sagar, N.A.; Vyas, N. Bioactive Compounds of Annona. In Bioactive Compounds in Underutilized Fruits and Nuts; Murthy, H.N., Bapat, V.A., Eds.; Springer Nature Switzerlad: Basel, Switzerland, 2019; pp. 1-26.

76. Lim, T.K. Edible Medicinal and Non-Medicinal Plants; Fruits; Springer Science+ Business Media: Dordrecht, The Netherlands; New York, NY, USA, 2012; Volume 1, 835p.

77. Andrade, E.H.A.; Zoghbi, M.D.G.B.; Maia, J.G.S.; Fabricius, H.; Marx, F. Chemical characterization of the fruit of Annona squamosa L. Occurring in the Amazon. J. Food Compos. Anal. 2001, 14, 227-232. [CrossRef]

78. Bala, S.; Nigam, V.K.; Tiwari, A.K.; Vidyarthi, A.S. Studies on nutraceutical properties of Annona squamosa. Appl. Biotechnol. Sustain. Dev. 2017, 81-87. [CrossRef]

79. Marroquín, A.L.; Cuevas, S.J.A.; Ramírez, D.G.; Reyes, L.; Reyes, C.A.; Reyes, T.B. Proximate composition, mineral nutrient and fatty acids of the seed of ilama, Annona diversifolia Saff. Sci. Res. Essays 2011, 6, 3089-3093. [CrossRef]

80. Quílez, A.M.; Fernández, A.M.A.; García, G.M.D.; De la Puerta, R. Potential therapeutic applications of the genus Annona: Local and traditional uses and pharmacology. J. Ethnopharmacol. 2018, 225, 244-270. [CrossRef]

81. Moghadamtousi, S.Z.; Fadaeinasab, M.; Nikzad, S.; Mohan, G.; Ali, H.M.; Kadir, H.A. Annona muricata (Annonaceae): A review of its traditional uses, isolated acetogenins and biological activities. Int. J. Mol. Sci. 2015, 16, 15625-15658. [CrossRef]

82. Coria, T.A.V.; Montalvo, G.E.; Yahia, E.M.; Obledo, V.E.N. Annona muricata: A comprehensive review on its traditional medicinal uses, phytochemicals, pharmacological activities, mechanisms of action and toxicity. Arab. J. Chem. 2018, 11, 662-691. [CrossRef]

83. Aguilar, H.G.; Zepeda, V.L.G.; García, M.M.D.L.; Vivar, V.M.D.L.Á.; Pérez, L.A.; Girón, P.M.I.; Coria, T.A.V.; Rodríguez, A.C.; Montalvo, G.E. Extraction of alkaloids using ultrasound from pulp and by-products of soursop fruit (Annona muricata L.). Appl. Sci. 2020, 10, 4869. [CrossRef]

84. Mannino, G.; Gentile, C.; Porcu, A.; Agliassa, C.; Caradonna, F.; Bertea, C.M. Chemical profile and biological activity of cherimoya (Annona cherimola Mill.) and atemoya (Annona atemoya) leaves. Molecules 2020, 25, 2612. [CrossRef] [PubMed]

85. Kumar, M.; Changan, S.; Tomar, M.; Prajapati, U.; Saurabh, V.; Hasan, M.; Sasi, M.; Maheshwari, C.; Singh, S.; Dhumal, S.; et al. Custard apple (Annona squamosa 1.) leaves: Nutritional composition, phytochemical profile, and health-promoting biological activities. Biomolecules 2021, 11, 614. [CrossRef]

86. Can, C.C.A.; Sauri, D.E.; Betancur, A.D.; Chel, G.L.; González, A.G.A.; Cuevas, G.L.F.; Pérez, P.E.; Moo, H.V.M. Tropical fruit peel powders as functional ingredients: Evaluation of their bioactive compounds and antioxidant activity. J. Funct. Foods 2017, 37, 501-506. [CrossRef]

87. Zahid, M.; Arif, M.; Rahman, M.A.; Singh, K.; Mujahid, M. Solvent extraction and gas chromatography-Mass spectrometry analysis of Annona squamosa L. Seeds for determination of bioactives, fatty acid/fatty oil composition, and antioxidant activity. J. Diet. Suppl. 2018, 15, 613-623. [CrossRef]

88. Schlie, G.M.A.; García, C.A.; González, E.A.R. In Vitro and In Vivo antiproliferative anticity of laherradurin and cherimolin-2 of Annona diversifolia Saff. Phytother. Res. 2009, 23, 1128-1133. [CrossRef] [PubMed]

89. Laguna, H.G.; Brechú, F.A.E.; De la Cruz, C.I.; González, E.A.R. A histochemical technique for the detection of annonaceous acetogenins. Methods Mol. Biol. 2017, 1560, 331-338. [CrossRef]

90. Jiménez, V.M.; Gruschwitz, M.; Schweiggert, R.M.; Carle, R.; Esquivel, P. Identification of phenolic compounds in soursop (Annona muricata) pulp by high-performance liquid chromatography with diode array and electrospray ionization mass spectrometric detection. Food Res. Int. 2014, 65, 42-46. [CrossRef]

91. Nawwar, M.; Ayoub, N.; Hussein, S.; Hashim, A.; El-Sharawy, R.; Wende, K.; Harms, M.; Lindequist, U. A flavonol triglycoside and investigation of the antioxidant and cell stimulating activities of Annona muricata Linn. Arch. Pharm. Res. 2012, 35, 761-767. [CrossRef]

92. George, V.C.; Kumar, N.D.R.; Rajkumar, V.; Suresh, P.K.; Ashok, K.R. Quantitative assessment of the relative antineoplastic potential of the n-butanolic leaf extract of Annona muricata Linn. in normal and immortalized human cell lines. Asian Pac. J. Cancer Prev. 2012, 13, 699-704. [CrossRef]

93. Santos, S.A.O.; Vilela, C.; Camacho, J.F.; Cordeiro, N.; Gouveia, M.; Freire, C.S.R.; Silvestre, A.J.D. Profiling of lipophilic and phenolic phytochemicals of four cultivars from cherimoya (Annona cherimola Mill.). Food Chem. 2016, 211, 845-852. [CrossRef]

94. Nandhakumar, E.; Indumathi, P. In Vitro antioxidant activities of methanol and aqueous extract of annona squamosa (L.) fruit pulp. JAMS J. Acupunct. Meridian Stud. 2013, 6, 142-148. [CrossRef] 
95. Panda, S.; Kar, A. Antidiabetic and antioxidative effects of Annona squamosa leaves are possibly mediated through quercetin-3-Oglucoside. BioFactors 2007, 31, 201-210. [CrossRef] [PubMed]

96. Shehata, M.G.; Abu-Serie, M.M.; Abd El-Aziz, N.M.; El-Sohaimy, S.A. Nutritional, phytochemical, and in vitro anticancer potential of sugar apple (Annona squamosa) fruits. Sci. Rep. 2021, 11, 6224. [CrossRef] [PubMed]

97. Solís, F.J.A.; Amador, H.C.; Hernández, M.M.R.; Durán, d.B.M.C. Caracterización fisicoquímica y comportamiento térmico del aceite de "almendra" de guanábana (Annona muricata, L.). Grasas Aceites 2010, 61, 58-66. [CrossRef]

98. George, V.C.; Kumar, D.R.N.; Suresh, P.K.; Kumar, R.A. Antioxidant, DNA protective efficacy and HPLC analysis of Annona muricata (soursop) extracts. J. Food Sci. Technol. 2015, 52, 2328-2335. [CrossRef] [PubMed]

99. Masson, L.; Camilo, C.; Gonzalez, K.; Caceres, A.; Jorge, N.; Torija, E.M. New sources of oilseeds from Latin American native fruits. Nat. Prod. Commun. 2008, 3, 357-362. [CrossRef]

100. García, S.P.; Verardo, V.; Gori, A.; Caboni, M.F.; Segura, C.A.; Fernández, G.A. Determination of lipid composition of the two principal cherimoya cultivars grown in Andalusian Region. LWT Food Sci. Technol. 2016, 65, 390-397. [CrossRef]

101. Chaoming, L.; Ninghua, T.; Yuping, L.; Huiling, L. Annomuricatin A, a new cyclopeptide from the seeds of Annona muricata. Acta Bot. Yunnanica 1995, 17, 459-462.

102. Wélé, A.; Zhang, Y.; Ndoye, I.; Brouard, J.P.; Pousset, J.L.; Bodo, B. A cytotoxic cyclic heptapeptide from the seeds of Annona cherimola. J. Nat. Prod. 2004, 67, 1577-1579. [CrossRef] [PubMed]

103. Wélé, A.; Ndoye, I.; Zhang, Y.; Brouard, J.P.; Bodo, B. Cherimolacyclopeptide D, a novel cycloheptapeptide from the seeds of Annona cherimola. Phytochemistry 2005, 66, 693-696. [CrossRef] [PubMed]

104. Dahiya, R.; Dahiya, S. Natural bioeffective cyclooligopeptides from plant seeds of Annona genus. Eur. J. Med. Chem. 2021, 214. [CrossRef] [PubMed]

105. Morita, H.; Sato, Y.; Kobayashi, J.N.I. Cyclosquamosins A-G, cyclic peptides from the seeds of Annona squamosa. Tetrahedron 1999, 55, 7509-7518. [CrossRef]

106. Morita, H.; Iizuka, T.; Choo, C.Y.; Chan, K.L.; Takeya, K.; Kobayashi, J. Vasorelaxant activity of cyclic peptide, cyclosquamosin B, from Annona squamosa. Bioorg. Med. Chem. Lett. 2006, 16, 4609-4611. [CrossRef]

107. Dellai, A.; Maricic, I.; Kumar, V.; Arutyunyan, S.; Bouraoui, A.; Nefzi, A. Parallel synthesis and anti-inflammatory activity of cyclic peptides cyclosquamosin D and Met-cherimolacyclopeptide B and their analogs. Bioorg. Med. Chem. Lett. 2010, 20, 5653-5657. [CrossRef]

108. Wu, P.; Wu, M.; Xu, L.; Xie, H.; Wei, X. Anti-Inflammatory cyclopeptides from exocarps of sugar-apples. Food Chem. 2014, 152, 23-28. [CrossRef]

109. Leboeuf, M.; Legueut, C.; Cavé, A.; Desconclois, J.; Forgacs, P.; Jacquemin, H. Alkaloids of annonaceae XXIX. Alkaloids of Annona muricata. Planta Med. 1981, 42, 37-44. [CrossRef]

110. Mohanty, S.; Hollinshead, J.; Jones, L.; Jones, P.W.; Thomas, D.; Watson, A.A.; Watson, D.G.; Gray, A.I.; Molyneuxc, R.J.; Nash, R.J. Annona muricata (Graviola): Toxic or therapeutic. Nat. Prod. Commun. 2008, 3, 31-33. [CrossRef]

111. Hasrat, J.A.; De Bruyne, T.; De Backer, J.P.; Vauquelin, G.; Vlietinck, A.J. Isoquinoline derivatives isolated from the fruit of Annona muricata as 5-HTergic 5-HT(1A) receptor agonists in rats: Unexploited anti-depressive (lad) products. J. Pharm. Pharmacol. 1997, 49, 1145-1149. [CrossRef]

112. Kotake, Y.; Okuda, K.; Kamizono, M.; Matsumoto, N.; Tanahashi, T.; Hara, H.; Caparros, L.D.; Ohta, S. Detection and determination of reticuline and N-methylcoculaurine in the Annonaceae family using liquid chromatography-tandem mass spectrometry. $J$. Chromatogr. B Anal. Technol. Biomed. Life Sci. 2004, 806, 75-78. [CrossRef] [PubMed]

113. Fofana, S.; Keita, A.; Balde, S.; Ziyaev, R.; Aripova, S.F. Alkaloids from leaves of Annona muricata. Chem. Nat. Compd. 2012, 48, 714 [CrossRef]

114. Matsushige, A.; Kotake, Y.; Matsunami, K.; Otsuka, H.; Ohta, S.; Takeda, Y. Annonamine, a new aporphine alkaloid from the leaves of Annona muricata. Chem. Pharm. Bull. 2012, 60, 257-259. [CrossRef]

115. Martínez, V.M.; De La Cueva, L.D.G.; Estrada, R.R.; González, L.N.M.; Ramírez, A.T.; Heinze, G. Bio-Guided isolation of the cytotoxic corytenchine and isocoreximine from roots of Annona cherimolia. Fitoterapia 2005, 76, 733-736. [CrossRef]

116. Vendramin, M.E.; Costa, E.V.; Pereira dos Santos, É.; Belém Pinheiro, M.L.; Barison, A.; Campos, F.R. Chemical constituents from the leaves of Annona rugulosa (Annonaceae). Biochem. Syst. Ecol. 2013, 49, 152-155. [CrossRef]

117. Lebrini, M.; Robert, F.; Roos, C. Inhibition effect of alkaloids extract from Annona squamosa plant on the corrosion of C38 steel in normal hydrochloric acid medium. Int. J. Electrochem. Sci. 2010, 5, 1698-1712.

118. Shami, A.M. The effect of alkaloidal fraction from Annona squamosa L. against pathogenic bacteria with antioxidant activities. Pharm. Sci. 2017, 24, 301-307. [CrossRef]

119. Pinto, N.C.C.; Silva, J.B.; Menegati, L.M.; Guedes, M.C.M.R.; Marques, L.B.; da Silva, T.P.; de Melo, R.C.N.; de Souza-Fagundes, E.M.; Salvador, M.J.; Scio, E. Cytotoxicity and bacterial membrane destabilization induced by Annona squamosa L. Extracts. An. Acad. Bras. Cienc. 2017, 89, 2053-2073. [CrossRef]

120. Johns, T.; Windust, A.; Jurgens, T.; Mansor, S.M. Antimalarial alkaloids isolated from Annona squamosa. Phytopharmacology 2011, 1, 49-53.

121. De La Cruz Chacón, I.; González, E.A.R. Liriodenine alkaloid in Annona diversifolia during early development. Nat. Prod. Res. 2012, 26, 42-49. [CrossRef] [PubMed] 
122. Orozco, C.J.A.; Cruz, O.R.; Martinez, V.M.; González, E.A.R. Aporphine alkaloid contents increase with moderate nitrogen supply in Annona diversifolia Saff. (Annonaceae) seedlings during diurnal periods. Nat. Prod. Res. 2016, 30, 2209-2214. [CrossRef] [PubMed]

123. Champy, P.; Melot, A.; Guérineau, V.; Gleye, C.; Fall, D.; Höglinger, G.U.; Ruberg, M.; Lannuzel, A.; Laprévote, O.; Laurens, A. Quantification of acetogenins in Annona muricata linked to atypical Parkinsonism in Guadeloupe. Mov. Disord. 2005, 20, 1629-1633. [CrossRef] [PubMed]

124. Champy, P.; Guérineau, V.; Laprévote, O. MALDI-TOF MS profiling of annonaceous acetogenins in Annona muricata products for human consumption. Molecules 2009, 14, 5235-5246. [CrossRef]

125. Melot, A.; Fall, D.; Gleye, C.; Champy, P. Apolar Annonaceous acetogenins from the fruit pulp of Annona muricata. Molecules 2009, 14, 4387-4395. [CrossRef]

126. Sun, S.; Liu, J.; Kadouh, H.; Sun, X.; Zhou, K. Three new antiproliferative Annonaceous acetogenins with mono-tetrahydrofuran ring from graviola fruit (Annona muricata). Bioorg. Med. Chem. Lett. 2014, 24, 2773-2776. [CrossRef]

127. Wu, F.E.; Gu, Z.M.; Zeng, L.; Zhao, G.X.; Zhang, Y.; McLaughlin, J.L.; Sastrodihardjo, S. Two new cytotoxic monotetrahydrofuran annonaceous acetogenins, annomuricins A and B, from the leaves of Annona muricata. J. Nat. Prod. 1995, 58, 830-836. [CrossRef]

128. Kim, G.S.; Zeng, L.; Alali, F.; Rogers, L.L.; Wu, F.E.; McLaughlin, J.L.; Sastrodihardjo, S. Two new mono-tetrahydrofuran ring acetogenins, annomuricin E and muricapentocin, from the leaves of Annona muricata. J. Nat. Prod. 1998, 61, 432-436. [CrossRef]

129. Liaw, C.C.; Chang, F.R.; Lin, C.Y.; Chou, C.J.; Chiu, H.F.; Wu, M.J.; Wu, Y.C. New cytotoxic monotetrahydrofuran annonaceous acetogenins from Annona muricata. J. Nat. Prod. 2002, 65, 470-475. [CrossRef]

130. Gleye, C.; Rafidiarison, N.; Duret, P.; Laurens, A.; Hocquemiller, R. Robustocin, a new acetogenin from the seeds of Annona muricata. Nat. Prod. Lett. 2000, 14, 239-245. [CrossRef]

131. Gromek, D.; Figadère, B.; Hocquemiller, R.; Cavé, A.; Cortes, D. Corepoxylone, a possible precursor of mono-tetrahydrofuran $\gamma$-lactone acetogenins: Biomimetic synthesis of corossolone. Tetrahedron 1993, 49, 5247-5252. [CrossRef]

132. Chang, F.R.; Wu, Y.C. Novel cytotoxic annonaceous acetogenins from Annona muricata. J. Nat. Prod. 2001, 64, 925-931. [CrossRef]

133. Yang, H.; Zhang, N.; Zeng, Q.; Yu, Q.; Ke, S.; Li, X. HPLC method for the simultaneous determination of ten annonaceous acetogenins after supercritical fluid $\mathrm{CO}_{2}$ extraction. Int. J. Biomed. Sci. 2010, 6, 202-207.

134. Ragasa, C.Y.; Soriano, G.; Torres, O.B.; Don, M.J.; Shen, C.C. Acetogenins from Annona muricata. Pharmacogn. J. 2012, 4, 32-37. [CrossRef]

135. Bonneau, N.; Schmitz-Afonso, I.; Brunelle, A.; Touboul, D.; Champy, P. Method development for quantification of the environmental neurotoxin annonacin in Rat plasma by UPLC-MS/MS and application to a pharmacokinetic study. J. Chromatogr. B Anal. Technol. Biomed. Life Sci. 2015, 1004, 46-52. [CrossRef] [PubMed]

136. Gutiérrez, M.T.; Durán, A.G.; Mejías, F.J.R.; Molinillo, J.M.G.; Megias, D.; Valdivia, M.M.; Macías, F.A. Bio-Guided isolation of acetogenins from Annona cherimola deciduous leaves: Production of nanocarriers to boost the bioavailability properties. Molecules 2020, 25, 4861. [CrossRef] [PubMed]

137. Kim, D.H.; Ma, E.S.; Suk, K.D.; Son, J.K.; Lee, J.S.; Woo, M.H. Annomolin and annocherimolin, new cytotoxic annonaceous acetogenins from Annona cherimolia seeds. J. Nat. Prod. 2001, 64, 502-506. [CrossRef] [PubMed]

138. Chen, C.Y.; Chang, F.R.; Chiu, H.F.; Wu, M.J.; Wu, Y.C. Aromin-A, an annonaceous acetogenin from Annona cherimola. Phytochemistry 1999, 51, 429-433. [CrossRef]

139. Mazahery, A.R.F.; Dator, R.P.; Concepcion, G.P.; Jacinto, S.D. Murihexocin C from the leaves of Annona squamosa Linn. Induces apoptosis in human colon carcinoma Col 2 cell line. Philipp. Agric. Sci. 2009, 92, 122-132. [CrossRef]

140. Araya, H.; Sahai, M.; Singh, S.; Singh, A.K.; Yoshida, M.; Hara, N.; Fujimoto, Y. Squamocin-O1 and squamocin-O2, new adjacent bis-tetrahydrofuran acetogenins from the seeds of Annona squamosa. Phytochemistry 2002, 61, 999-1004. [CrossRef]

141. Chen, Y.; Chen, J.W.; Li, X. Monotetrahydrofuran annonaceous acetogenins from the seeds of Annona squamosa. Phytochem. Lett. 2012, 5, 33-36. [CrossRef]

142. Miao, Y.; Xu, X.; Yuan, F.; Shi, Y.; Chen, Y.; Chen, J.; Li, X. Four cytotoxic annonaceous acetogenins from the seeds of Annona squamosa. Nat. Prod. Res. 2016, 30, 1273-1279. [CrossRef]

143. Hopp, D.C.; Alali, F.Q.; Gu, Z.M.; McLaughlin, J.L. Three new bioactive bis-adjacent THF-ring acetogenins from the bark of Annona squamosa. Bioorg. Med. Chem. 1998, 6, 569-575. [CrossRef]

144. Protais, P.; Arbaoui, J.; Bakkali, E.H.; Bermejo, A.; Cortes, D. Effects of various isoquinoline alkaloids on in vitro 3h-dopamine uptake by rat striatal synaptosomes. J. Nat. Prod. 1995, 58, 1475-1484. [CrossRef]

145. Neske, A.; Ruiz, H.J.; Cabedo, N.; Cortes, D. Acetogenins from Annonaceae family. Their potential biological applications. Phytochemistry 2020, 174, 112332. [CrossRef]

146. Anaya, E.L.M.; García, M.M.d.L.; Domínguez, Á.A.J.; Yahia, E.M.; Salazar, L.N.J.; González, A.G.A.; Montalvo, G.E. Annonas: Underutilized species as a potential source of bioactive compounds. Food Res. Int. 2020, 138, 109775. [CrossRef] [PubMed]

147. Roduan, M.R.; Abd Hamid, R.; Mohtarrudin, N. Modulation of cancer signalling pathway(s) in two -stage mouse skin tumorigenesis by annonacin. BMC Complement. Altern. Med. 2019, 19, 238. [CrossRef]

148. Ogbu, P.N.; Ugota, E.O.; Onwuka, R.U.; Ogbu, I.M.; Aloke, C. Effect of acetogenin fraction of Annona muricata leaves on antioxidant status and some indices of benign prostatic hyperplasia in rats. Redox Rep. 2020, 25, 80-86. [CrossRef]

149. Agu, K.C.; Okolie, N.P.; Falodun, A.; Engel-Lutz, N. In Vitro anticancer assessments of Annona muricata fractions and In Vitro antioxidant profile of fractions and isolated acetogenin (15-acetyl guanacone). J. Cancer Res. Pract. 2018, 5, 53-66. [CrossRef] 
150. Foster, K.; Oyenihi, O.; Rademan, S.; Erhabor, J.; Matsabisa, M.; Barker, J.; Langat, M.K.; Kendal-Smith, A.; Asemota, H.; Delgoda, R. Selective cytotoxic and anti-metastatic activity in DU-145 prostate cancer cells induced by Annona muricata L. bark extract and phytochemical, annonacin. BMC Complement. Med. Ther. 2020, 20, 375. [CrossRef]

151. Fuel, M.; Mesas, C.; Martínez, R.; Ortiz, R. Antioxidant and antiproliferative potential of ethanolic extracts from Moringa oleifera, Tropaeolum tuberosum and Annona cherimola in colorrectal cancer cells. Biomed. Pharmacother. 2021, 143, 112248. [CrossRef]

152. Younes, M.; Ammoury, C.; Haykal, T.; Nasr, L.; Sarkis, R.; Rizk, S. The selective anti-proliferative and pro-apoptotic effect of $A$. cherimola on MDA-MB-231 breast cancer cell line. BMC Complement. Med. Ther. 2020, 20, 343. [CrossRef]

153. Florence, N.T.; Benoit, M.Z.; Jonas, K.; Alexandra, T.; Désiré, D.D.P.; Pierre, K.; Théophile, D. Antidiabetic and antioxidant effects of Annona muricata (Annonaceae), aqueous extract on streptozotocin-induced diabetic rats. J. Ethnopharmacol. 2014, 151, 784-790. [CrossRef]

154. Alsenosy, A.W.A.; El-Far, A.H.; Sadek, K.M.; Ibrahim, S.A.; Atta, M.S.; Sayed-Ahmed, A.; Al Jaouni, S.K.; Mousa, S.A. Graviola (Annona muricata) attenuates behavioural alterations and testicular oxidative stress induced by streptozotocin in diabetic rats. PLoS ONE 2019, 14, e0222410. [CrossRef] [PubMed]

155. Ahalya, B.; Shankar, K.R.; Kiranmayi, G.V.N. Exploration of anti-hyperglycemic and hypolipidemic activities of ethanolic extract of Annona muricata bark in alloxan induced diabetic rats. Int. J. Pharm. Sci. Rev. Res. 2014, 25, 21-27.

156. Calzada, F.; Solares, P.J.I.; Ordoñez, R.R.M.; Velazquez, C.; Barbosa, E.; García, H.N.; Mendez, L.D.; Correa, B.J. Antihyperglycemic activity of the leaves from Annona cherimola miller and rutin on alloxan-induced diabetic rats. Pharmacognosy Res. 2017, 9, 1-6. [CrossRef] [PubMed]

157. Martínez, S.J.; Calzada, F.; Barbosa, E.; Valdés, M. Antihyperglycemic and antilipidemic properties of a tea infusion of the leaves from Annona cherimola miller on streptozocin-induced type 2 diabetic mice. Molecules 2021, 26, 2408. [CrossRef]

158. Kaleem, M.; Medha, P.; Ahmed, Q.U.; Asif, M.; Bano, B. Beneficial effects of Annona squamosa extract in streptozotocin-induced diabetic rats. Singap. Med. J. 2008, 49, 800-804. [PubMed]

159. Basha, S.K.H.; Subramanian, S. Biochemical evaluation of antidiabetic and antioxidant potentials of Annona squamosa leaves extracts studied in STZ-induced diabetic rats. Int. J. Pharm. Sci. Res. 2011, 2, 643-655.

160. Mohd, M.; Alam, K.S.; Mohd, A.; Abhishek, M.; Aftab, A. Antidaiabetic activity of the aqueous extract o Annona squamosa in streptozotocin induced-hyperglycemic rats. Pharma Res. 2009, 2, 59-63.

161. Sahu, M.; Nk, S.; Alagarsamy, V.; Bp, R. Comparative Evaluation of Antidiabetic and Antioxidant Activities of Aqueous Fruit Peel and Leaf Extracts of Annona Squamosa on High Fat Diet and Multiple Low Dose Streptozotocin Mouse Model of Diabetes. 2016. Available online: https:/ / austinpublishinggroup.com/pharmacology-therapeutics/fulltext/ajpt-v4-id1081.php (accessed on 17 October 2021).

162. Calzada, F.; Valdes, M.; Garcia, H.N.; Velázquez, C.; Barbosa, E.; Bustos, B.C.; Quijano, L.; Pina, J.E.; Mendieta, W.J.E. Antihyperglycemic activity of the leaves from Annona diversifolia Safford. and farnesol on normal and alloxan-induced diabetic mice. Pharmacogn. Mag. 2019, 15, 5. [CrossRef]

163. Brindis, F.; González, T.M.E.; González, A.M.; Aguirre, H.E.; Villalobos, M.R. Aqueous extract of Annona macroprophyllata: A potential $\alpha$-glucosidase inhibitor. Biomed. Res. Int. 2013, 2013, 591313. [CrossRef] [PubMed]

164. Ishola, I.O.; Awodele, O.; Olusayero, A.M.; Ochieng, C.O. Mechanisms of analgesic and anti-inflammatory properties of Annona muricata Linn. (Annonaceae) fruit extract in rodents. J. Med. Food 2014, 17, 1375-1382. [CrossRef] [PubMed]

165. Bitar, R.M.; Fahmi, R.R.; Borjac, J.M. Annona muricata extract reduces inflammation via inactivation of NALP3 inflammasome. J. Nat. Remedies 2019, 19, 12-23. [CrossRef]

166. Ayun, N.Q.; Kusmardi; Nurhuda; Elya, B. Anti-inflammation of soursop leaves (Annona muricata L.) against hemorrhoids in mice induced by croton oil. Pharmacogn. J. 2020, 12, 784-792. [CrossRef]

167. Silva, H.N.; Rabelo, S.V.; Diniz, T.C.; Oliveira, F.G.S.; Teles, R.B.A.; Silva, J.C.; e Silva, M.G.; Coutinho, H.D.M.; de Menezes, I.R.A.; Almeida, J.R.G.S. Antinociceptive and anti-inflammatory activities of ethanolic extract from atemoya (Annona cherimola Mill $\times$ Annona squamosa L.). Afr. J. Pharm. Pharmacol. 2017, 11, 224-232. [CrossRef]

168. Hemalatha, K.; Satyanarayana, D. Anti-Inflammatory activity of Annona squamosa Linn. Biomed. Pharmacol. J. 2009, 2, 17-20.

169. Chavan, M.J.; Wakte, P.S.; Shinde, D.B. Analgesic and anti-inflammatory activity of Caryophyllene oxide from Annona squamosa L. bark. Phytomedicine 2010, 17, 149-151. [CrossRef]

170. Chavan, M.J.; Wakte, P.S.; Shinde, D.B. Analgesic and anti-inflammatory activities of 18-acetoxy-ent-kaur-16-ene from Annona squamosa L. bark. Inflammopharmacology 2011, 19, 111-115. [CrossRef]

171. Pareek, S.; Yahia, E.M.; Pareek, O.P.; Kaushik, R.A. Postharvest physiology and technology of Annona fruits. Food Res. Int. 2011, 44, 1741-1751. [CrossRef]

172. Anaya, D.J.; Hernández, O.M.; Tafolla, A.J.; Báez, S.R.; Gutiérrez, M.P.; Tiznado, H.M. Estudios Sociales. Rev. Aliment. Contemp. Desarro. Reg. 2021, 31, 291.

173. Tovar, G.B.; De Oca, M.M.; García, G.H.S.; Montalvo, G.E. Efecto de emulsiones de cera y 1-Metilciclopropeno en la conservación poscosecha de guanabana. Rev. Chapingo Ser. Hortic. 2011, 17, 53-61.

174. Verdugo, F.A.; Peñuelas, R.O.; Argentel, M.L.; Leyva, P.J.A.; González, A.J. Algunas consideraciones sobre el manejo poscosecha de la zanahoria. Acta Agríc. Pecu. 2021, 7. Available online: http://aap.uaem.mx/index.php/aap/article/view/184 (accessed on 17 October 2021). 
175. Tiencheu, B.; Egbe, C.A.; Achidi, U.A.; Tenyang, N.; Tiepma, N.E.F.; Djikeng, F.T.; Fossi, T.B. Nutritional, organoleptic and phytochemical properties of soursop (Annona muricata) pulp and juice after postharvest ripening. Eur. J. Nutr. Food Saf. 2021, 13, 15-28. [CrossRef]

176. Morton, J.F. Fruits of Warm Climates, 1st ed.; Echo Point Books and Media: Miami, FL, USA, 1987.

177. Jiménez, Z.J.O.; Balois, M.R.; Alia, T.I.; Juárez, L.P.; Jiménez, R.E.I.; Sumaya, M.M.T.; Bello, L.J.E. Tópicos del manejo poscosecha del fruto de guanábana (Annona muricata L.). Rev. Mex. Cienc. Agric. 2017, 8, 1155-1167. [CrossRef]

178. Balaguera, L.H.E.; Salamanca, G.F.A.; García, J.C.; Herrera, Á.A. Etileno y retardantes de la maduración en la poscosecha de productos agrícolas. Una revisión. Rev. Colomb. Cienc. Hortíc. 2015, 8, 302-313. [CrossRef]

179. Bapat, V.A.; Trivedi, P.K.; Ghosh, A.; Sane, V.A.; Ganapathi, T.R.; Nath, P. Ripening of fleshy fruit: Molecular insight and the role of ethylene. Biotechnol. Adv. 2010, 28, 94-107. [CrossRef]

180. Wijesinghe, M.G.P.; Kumara, K.G.D.; Kumara, P.J.B.D.A. Effect of 1-Methylcyclopropene on shelf-life and postharvest qualities of four Annona species in Sri Lanka. J. Food Agric. 2021, 14, 1-17. [CrossRef]

181. Montalvo, G.E.; Fernández, L.A.E.; Paez, H.R.; De Oca, M.M.; Gómez, T.B. Uso combinado de 1-meticiclopropeno y emulsiones de cera en la conservación de guanábana (Annona muricata). Rev. Bras. Frutic. 2014, 36, $296-304$.

182. Cortés, R.J.H.; González, E.; Estrada, J.Á.; Huerta, O.; Blancas, B.F.J. Evaluación de quitosano comercial y extractos acuosos de mesocarpio de coco. TIP Rev. Espec. Cienc. Quim.-Biol. 2021, 24, 1-11.

183. Gómez, J.R.; Villarreal, B.T.; Vásquez, L.A.; Arteaga, G.I.A.; Osuna, G.J.A. Actividad esporicida de la solución electrolizada con ph neutro en hongos de importancia postcosecha. Rev. Mex. Cienc. Agríc. 2017, 19, 3993-4007. [CrossRef]

184. López, V.A.; De La Cruz, M.J.; León, G.E.; García, G.S.H.; Vázquez, H.V.M. Aplicación de tratamientos hidrotérmico, fungicida y cera sobre el oscurecimiento superficial en guanábana. Rev. Mex. Cienc. Agríc. 2018, 9, 1075-1081. [CrossRef]

185. De los Santos, S.M.A.; Balois, M.R.; Jiménez, Z.J.O.; Alia, T.I.; López, G.G.G.; Palomino, H.Y.A.; Berumen, V.G.; García, P.J.D. Edible coating based on roselle (Hibiscus sabdariffa L.) mucilage applied to soursop fruits in postharvest storage. J. Food Qual. 2020, 2020, 12. [CrossRef]

186. Siang, M.L.; Ding, P.; Mohamed, M.T.M. Response of 1-Methycyclopropene on postharvest quality of local soursop (Annona muricata L.). Sains Malays. 2019, 48, 571-579.

187. Ramos, G.A.; González, E.E.; Romanazzi, G.; Landi, L.; Gutiérrez, M.P. Effects of chitosan in the control of postharvest anthracnose of soursop (Annona muricata) Fruit. Rev. Mex. Ing. Quim. 2020, 19, 99-108. [CrossRef]

188. Minh, P.N.; Vo, S.T.; Tram, N.D.; Nga, H.N.; Bang, T.N.; Trinh, D.M.T. Application of chitosan edible coating for soursop (Annona muricata) storage. J. Pharm. Sci. Res. 2019, 11, 284-288.

189. Jiménez, Z.J.O.; Balois, M.R.; Alia, T.I.; Sánchez, H.L.M.; Jiménez, R.E.I.; Bello, L.J.E.; García, P.J.D.; Juárez, L.P. Cold storage of two selections of soursop (Annona muricata L.) in Nayarit, Mexico. J. Food Qual. 2017, 2017, 4517469. [CrossRef]

190. Guardado, V.L.; Tovar, P.E.; Chacón, L.A.; López, G.U.; Gutiérrez, M.P.; Stoll, A.; Aguilera, S. Identification and characterization of a new Bacillus atrophaeus strain B5 as biocontrol agent of postharvest anthracnose disease in soursop (Annona muricata) and avocado (Persea americana). Microbiol. Res. 2018, 210, 26-32. [CrossRef] 